\title{
DELTA-SHOCKS AS LIMITS OF VANISHING VISCOSITY FOR MULTIDIMENSIONAL ZERO-PRESSURE GAS DYNAMICS
}

\author{
$\mathrm{BY}$ \\ JIEQUAN LI (Institute of Mathematics, Academia Sinica, Beijing, 100080, China) \\ AND \\ HANCHUN YANG (Dept. of Mathematics, Yunnan University, Kunming, 650091, China and \\ Institute of Mathematics, Academia Sinica, Beijing, 100080, China)
}

\begin{abstract}
In this paper we study zero-pressure gas dynamics, which is a nonstrict hyperbolic system of nonlinear conservation laws with delta-shock waves in solutions. By using the generalized Rankine-Hugoniot relations to solve the Riemann problem with two pieces of constant initial data, multidimensional planar delta-shock waves dependent upon a family of one parameter are obtained. Furthermore, we choose a unique entropy solution through the process of a viscosity vanishing, and obtain a stability for deltashocks in multidimensions.
\end{abstract}

1. Introduction. In this paper we consider the multidimensional zero-pressure flow in gas dynamics which is described by

$$
\left\{\begin{array}{l}
\rho_{t}+\nabla \cdot(\rho U)=0, \\
(\rho U)_{t}+\nabla \cdot(\rho U \otimes U)=O,
\end{array}\right.
$$

where $\rho(t, X) \geq 0\left(X=\left(x_{1}, x_{2}, \ldots, x_{n}\right) \in \mathbb{R}^{n}\right)$ and $U=\left(u_{1}, u_{2}, \ldots, u_{n}\right)$ are the density and velocity, respectively; $\nabla=\left(\partial x_{1}, \partial x_{2}, \ldots, \partial x_{n}\right)$ is the gradient operator with respect to the space variables, $\otimes$ is the usual tensor product of two vectors. Here and after, denote $O=(0,0, \ldots, 0)$ by the $n$-D null vector. The model has a number of origins, such as the flux-splitting numerical schemes [LC, AH], adhesion particle dynamics [SZe, ERS], zero-pressure isentropic gas dynamics $[\mathrm{B}, \mathrm{BG}]$, and the electron-sheet evolution [M-Z], etc. The system (1.1) has an $(n+1)$-fold eigenvalue $\lambda=U \cdot N$ in the direction $N=$ $\left(\alpha_{1}, \alpha_{2}, \ldots, \alpha_{n}\right)$ satisfying $|N|^{2}=\sum_{i=1}^{n} \alpha_{i}^{2}=1$ associated with an incomplete set of right eigenvectors. Thus, the system is nonstrictly hyperbolic and not diagonalizable in $\rho>0$.

In earlier papers [SZh, LZ], Sheng, Zhang and $\mathrm{Li}$ solved the 1-D and 2-D Riemann problems for (1.1) completely. A distinctive feature is that delta-shocks instead of shocks

Received June 21, 1999.

2000 Mathematics Subject Classification. Primary 34C05, 35B05, 35B35, 35M20, 35Q35.

Key words and phrases. Multidimensional zero-pressure gas dynamics, delta-shock, vacuum, Riemann problem, stability, viscosity vanishing. 
develop in solutions of (1.1). The delta-shock is characterized by the location, propagation speed and the weight which represents the mass of concentrated particles. Here we would like to mention the adhesion particle dynamics in astrophysics, which can be used to describe the motion of free particles sticking under collision in low temperature and explain the formation of large-scale structures in the universe. Therefore, the delta-shock is interpreted as the galaxies in the universe, or the concentration of particles.

A delta-shock wave is a kind of discontinuity on which at least one of the state variables becomes a singular measure in the form of a weighted Dirac delta function in solutions. It was independently discovered by Tan and Zhang [TZ] in 1990 when they studied the Riemann problem of two-dimensional Burgers-type equations

$$
U_{t}+\nabla \cdot(U \otimes U)=O
$$

For certain values of initial data, delta-shock waves were found necessary and used as parts in their solutions. Then, Tan, Zhang and Zheng [TZZ] established the existence, uniqueness and stability of delta-shock waves to some viscous perturbations in the reduced one-dimensional case. In addition, we also refer the reader to papers [Ko, Le, KK, J, SZh, LZ, LL] for more details.

Naturally, the simplest 2-D Riemann problem consists of the two pieces of initial data

$$
(\rho, U)(0, X)= \begin{cases}\left(\rho_{1}, U_{1}\right), & X \cdot N<0, \\ \left(\rho_{2}, U_{2}\right), & X \cdot N>0,\end{cases}
$$

where the straight line $\Gamma: \alpha_{1} x_{1}+\alpha_{2} x_{2}=0$ divides the $\left(x_{1}, x_{2}\right)$-plane into two parts $\Omega_{1}=\{X \mid X \cdot N<0\}$ and $\Omega_{2}=\{X \mid X \cdot N>0\}$, and the normal $N=\left(\alpha_{1}, \alpha_{2}\right)$ of $\Gamma$ with $\alpha_{1}^{2}+\alpha_{2}^{2}=1$ points from $\Omega_{1}$ towards $\Omega_{2}$. We let $\left(\rho, U ; \sigma, w, U_{\delta}\right)$ denote the delta-shock solution of (1.1) and (1.2), i.e., a solution of the form

$$
(\rho, U)(t, X)= \begin{cases}\left(\rho_{1}, U_{1}\right), & X \cdot N-\sigma t<0 \\ \left(w(t) \delta(X \cdot N-\sigma t), U_{\delta}\right), & X \cdot N-\sigma t=0 \\ \left(\rho_{2}, U_{2}\right), & X \cdot N-\sigma t>0\end{cases}
$$

where $\delta(x)$ is a Dirac delta function, the propagation speed and weight are

$$
\sigma=U_{\delta} \cdot N \in\left(U_{2} \cdot N, U_{1} \cdot N\right)
$$

and

$$
w(t)=\sqrt{\rho_{1} \rho_{2}}([U] \cdot N) t
$$

respectively, and

$$
U_{\delta}=\frac{\sqrt{\rho_{1}} U_{1}+\sqrt{\rho_{2}} U_{2}}{\sqrt{\rho_{1}}+\sqrt{\rho_{2}}} .
$$

Here $U_{i}=\left(u_{1 i}, u_{2 i}\right), i=1,2 ; U_{\delta}=\left(u_{1 \delta}, u_{2 \delta}\right) ;[G]=G_{1}-G_{2}$, etc. The results can also be obtained directly from [LL].

In Sec. 2, we solve the 3-D Riemann problem with two constant states separated by a plane and obtain the delta-shock solution defined in (1.3-1.6) in which (1.5) is replaced 
by

$$
w(t)=\alpha_{1} \sqrt{\rho_{1} \rho_{2}}([U] \cdot N) t,
$$

where $\alpha_{1}>0$, and $N=\left(\alpha_{1}, \alpha_{2}, \alpha_{3}\right)$ is the normal of the plane $\Gamma: \alpha_{1} x_{1}+\alpha_{2} x_{2}+\alpha_{3} x_{2}=0$. Furthermore, we can also obtain the similar results for the $n$-D $(n>3)$ case.

From (1.5) and (1.7), we conclude that the weight depends actually on the length of the normal $N$ for $n \geq 2$, especially on a family of one parameter as $n \geq 3$. In view of this, it is worthy to study the following system:

$$
\left\{\begin{array}{l}
\rho_{t}+\nabla \cdot(\rho U)=0, \\
(\rho U)_{t}+\nabla \cdot(\rho U \otimes U)=\varepsilon t \Delta U,
\end{array}\right.
$$

where a relevant viscosity is considered, and $\Delta$ is the Laplace operator. We prove rigorously that our Riemann solutions including the delta-shock are weak star limits of $\left(\rho^{\varepsilon}, U^{\varepsilon}\right)$ as $\varepsilon \rightarrow 0^{+}$, and the limits do not depend upon the choice for $N$. Here $\left(\rho^{\varepsilon}, U^{\varepsilon}\right)$ are the self-similar solutions of the corresponding Riemann problem for the system (1.8). We emphasize that the delta-shock as the limit of solutions to (1.8) is exactly a solution of (1.1), which is just desired to choose among the delta-shocks dependent upon a family of one parameter. Thus, we establish the stability for delta-shocks of the zero-pressure flow in multidimensions. These are the contents of Sections 3 and 4 .

It is noticed that, for the system (1.1), [SZh] studied the viscous system (1.8) when $n=1$ and obtained similar results. When $n=2$, they discussed the so-called pseudosteady self-similar viscous system and obtained an unfitable weight of the delta-shock. However, their work is no doubt the pioneering and excellent one. In the present work, we extend the $1-\mathrm{D}$ results to those in $n(n \geq 2)$ dimensions. Indeed, Sec. 2 does not work for $n=1$ at all. Sections 3 and 4 basically adapt the same arguments of the 1-D case from [SZh] to $n \geq 2$ with a great modification. Besides, we also explore formulating explicitly a general frame for delta-shocks in multidimensional systems.

2. The Riemann problem with two pieces of initial data. By using the generalized Rankine-Hugoniot relations, this section deals with the basic Riemann problem with two constant states separated by a hyperplane, especially a straight line in two dimensions and a plane in three dimensions. As a special case for $n=2$, it can be solved in the same way as $n=3$, or see [LL]. In what follows, we mainly consider the 3 -D case.

Let the plane $\Gamma: \alpha_{1} x_{1}+\alpha_{2} x_{2}+\alpha_{3} x_{2}=0$ divide $\left(x_{1}, x_{2}, x_{3}\right)$-space into two infinite parts $\Omega_{1}$ and $\Omega_{2}$, and orient the normal $N=\left(\alpha_{1}, \alpha_{2}, \alpha_{3}\right)$ of $\Gamma$ with $\alpha_{1}^{2}+\alpha_{2}^{2}+\alpha_{3}^{2}=1$ from $\Omega_{1}$ towards $\Omega_{2}$. The initial data takes the form

$$
(\rho, U)(0, X)= \begin{cases}\left(\rho_{1}, U_{1}\right), & X \cdot N<0, \\ \left(\rho_{2}, U_{2}\right), & X \cdot N>0\end{cases}
$$

where $\rho_{1}, \rho_{2}>0$. We have the following lemma.

LEMMA 2.1. The necessary and sufficient condition of the overlap of characteristics on both sides of the initial discontinuity $\Gamma$ is

$$
[U] \cdot N>0 \text {. }
$$


Proof. Since the characteristic hyperplanes through $\Gamma$ defined by the eigenvalues $U_{1} \cdot N$ and $U_{2} \cdot N$ are respectively

$$
\begin{aligned}
& \pi_{1}: X \cdot N=\left(U_{1} \cdot N\right) t, \\
& \pi_{2}: X \cdot N=\left(U_{2} \cdot N\right) t,
\end{aligned}
$$

it follows from (2.2) that

$$
\left(X_{1}-X_{2}\right) \cdot N>0 \text {, }
$$

where $\left(t, X_{1}\right)$ is the point on $\pi_{1}$ and $\left(t, X_{2}\right)$ the point on $\pi_{2}$. This completes the proof.

Lemma 2.1 shows that the delta-shock must emit from the initial discontinuity $\Gamma$ when (2.2) is satisfied. To solve the Riemann problem (1.1) and (2.1), we first give the definition of a measure solution to (1.1) and introduce the generalized Rankine-Hugoniot relation in three dimensions.

Denote by $B M\left(\mathbb{R}^{n}\right)$ the space of bounded Borel measures on $\mathbb{R}^{n}$. Consider a mass distribution $\rho$ and a momentum distribution $Q$,

$$
\begin{aligned}
& \rho \in C\left([0, \infty), B M\left(\mathbb{R}^{n}\right)\right), \quad \rho \geq 0, \\
& Q \in\left(C\left([0, \infty), B M\left(\mathbb{R}^{n}\right)\right)\right)^{n},
\end{aligned}
$$

such that

$$
|Q(t, \cdot)| \leq M \rho(t, \cdot), \quad t \in[0, \infty)
$$

holds in the sense of measures, where $M$ is a constant. By Radon-Nykodým's theorem, for all $t \in[0, \infty)$, there exists a velocity $U(t, \cdot) \in\left(L^{\infty}(\rho(t, \cdot))\right)^{n}$, and $|U| \leq M$ such that

$$
Q(t, X)=U(t, X) \rho(t, X) .
$$

Then we define $\rho S(U) \in D^{\prime}\left([0, \infty) \times \mathbb{R}^{n}\right)$ by

$$
\langle\rho S(U), \phi\rangle=\int_{0}^{\infty} d t \int_{\mathbb{R}^{n}} S(U)(t, X) \phi(t, X) d \rho, \quad \phi \in C_{0}^{\infty}\left([0, \infty) \times \mathbb{R}^{n}\right)
$$

where $S: \mathbb{R}^{n} \rightarrow \mathbb{R}^{n}$ is a $C^{1}$ function. Thus we can give the definition of a solution to (1.1) in the sense of measures in arbitrary space dimensions.

Definition 2.1. Let $(\rho, U)$ satisfy $(2.3-2.5)$. Then $(\rho, U)$ is a solution of $(1.1)$ in the sense of measures if

$$
\begin{aligned}
\int_{0}^{\infty} \int_{\mathbb{R}^{n}}\left(\phi_{t}+U \cdot \nabla \phi\right) d \rho d t & =0 \\
\int_{0}^{\infty} \int_{\mathbb{R}^{n}} U\left(\phi_{t}+U \cdot \nabla \phi\right) d \rho d t & =O,
\end{aligned}
$$

hold for all $\phi \in C_{0}^{\infty}\left([0, \infty) \times \mathbb{R}^{n}\right)$.

REMARKs. (1). Since $U(t, \cdot)$ is defined by $\rho(t, \cdot)$ almost everywhere, we require at least that

$$
t \rightarrow \int_{\mathbb{R}^{n}} \phi(X) U(t, X) d \rho, \quad \phi \in C_{0}^{\infty}\left(\mathbb{R}^{n}\right)
$$

is measurable.

(2). The continuity conditions in $C\left([0, \infty), B M\left(\mathbb{R}^{n}\right)\right)$ may be used to give an interpretation for $(\rho, U)$ to take on initial values. 
DEFINITION 2.2. An $(n+1)$-dimensional weighted delta function $w(s) \delta_{\Sigma}$ supported on a smooth surface $\Sigma$ parametrized as $t=t(s), X=X(s)\left(s \in \mathbb{R}_{+}^{n}\right)$ is defined by

$$
\left\langle w(s) \delta_{L}, \phi(t, X)\right\rangle=\int_{\mathbb{R}_{+}^{n}} w(s) \phi(t(s), X(s)) d s
$$

for all $\phi \in C_{0}^{\infty}\left(\mathbb{R}_{+}^{n+1}\right)$.

Note that in this definition a surface is replaced by a curve when $n=1$. See [TZ, SZh, LL], etc.

Now suppose that a smooth discontinuity surface $\Sigma$ of a parametrized form $X=$ $X(t, s)$ separates $(t, X)$-space into two infinite parts $\Omega_{1}$ and $\Omega_{2}$, and a delta-shock solution of (1.1) takes the form

$$
(\rho, U)(t, X)= \begin{cases}\left(\rho_{1}, U_{1}\right)(t, X), & (t, X) \in \Omega_{1} \\ \left(w(t, s) \delta(t, X-X(t, s)), U_{\delta}(t, s)\right), & (t, X) \in \Sigma \\ \left(\rho_{2}, U_{2}\right)(t, X), & (t, X) \in \Omega_{2}\end{cases}
$$

Then it is the solution of (1.1) in the sense of Definition 2.1 if the generalized RankineHugoniot relation

$$
\begin{aligned}
\frac{\partial X}{\partial t} & =U_{\delta}(t, s), \\
\frac{\partial w}{\partial t} & =([\rho],[\rho U]) \cdot\left(N_{t}, N_{X}\right), \\
\frac{\partial\left(w U_{\delta}\right)}{\partial t} & =([\rho U],[\rho U \otimes U]) \cdot\left(N_{t}, N_{X}\right)
\end{aligned}
$$

is satisfied, where $\left(\rho_{1}, U_{1}\right)$ and $\left(\rho_{2}, U_{2}\right)(t, X)$ are smooth solutions of (1.1) in $\Omega_{1}$ and $\Omega_{2}$ respectively, $s=\left(s_{1}, s_{2}\right) \in[0, \infty) \times[0, \infty)$ are suitable parameters, and $\left(N_{t}, N_{X}\right)=$ $\left(N_{t}, N_{x_{1}}, N_{x_{2}}, N_{x_{3}}\right)=$

$$
\left(-\left|\begin{array}{lll}
u_{1 \delta} & u_{2 \delta} & u_{3 \delta} \\
\frac{\partial x_{1}}{\partial s_{1}} & \frac{\partial x_{2}}{\partial s_{1}} & \frac{\partial x_{3}}{\partial s_{1}} \\
\frac{\partial x_{1}}{\partial s_{2}} & \frac{\partial x_{2}}{\partial s_{2}} & \frac{\partial x_{3}}{\partial s_{2}}
\end{array}\right|,\left|\begin{array}{ll}
\frac{\partial x_{2}}{\partial s_{1}} & \frac{\partial x_{3}}{\partial s_{1}} \\
\frac{\partial x_{2}}{\partial s_{2}} & \frac{\partial x_{3}}{\partial s_{2}}
\end{array}\right|,-\left|\begin{array}{ll}
\frac{\partial x_{1}}{\partial s_{1}} & \frac{\partial x_{3}}{\partial s_{1}} \\
\frac{\partial x_{1}}{\partial s_{2}} & \frac{\partial x_{3}}{\partial s_{2}}
\end{array}\right|,\left|\begin{array}{ll}
\frac{\partial x_{1}}{\partial s_{1}} & \frac{\partial x_{2}}{\partial s_{1}} \\
\frac{\partial x_{1}}{\partial s_{2}} & \frac{\partial x_{2}}{\partial s_{2}}
\end{array}\right|\right)
$$

is the normal of $\Sigma$ postulated to orient from $\Omega_{1}$ towards $\Omega_{2}$ (see [LZ]).

Introduce the notation

$$
\left[U_{1}, U_{2}, \frac{\partial X}{\partial s}\right]=\left|\begin{array}{cccc}
u_{11} & u_{12} & \frac{\partial x_{1}}{\partial s_{1}} & \frac{\partial x_{1}}{\partial s_{2}} \\
u_{21} & u_{22} & \frac{\partial x_{2}}{\partial s_{1}} & \frac{\partial x_{2}}{\partial s_{2}} \\
u_{31} & u_{32} & \frac{\partial x_{3}}{\partial s_{1}} & \frac{\partial x_{3}}{\partial s_{2}} \\
1 & 1 & 0 & 0
\end{array}\right|
$$


Then the generalized Rankine-Hugoniot relation (2.11) can be rewritten in the form

$$
\begin{aligned}
\frac{\partial X}{\partial t} & =U_{\delta}(t, s) \\
\frac{\partial w}{\partial t} & =\rho_{1}\left[U_{1}, U_{\delta}, \frac{\partial X}{\partial s}\right]-\rho_{2}\left[U_{2}, U_{\delta}, \frac{\partial X}{\partial S}\right], \\
\frac{\partial\left(w U_{\delta}\right)}{\partial t} & =\rho_{1} U_{1}\left[U_{1}, U_{\delta}, \frac{\partial X}{\partial s}\right]-\rho_{2} U_{2}\left[U_{2}, U_{\delta}, \frac{\partial X}{\partial s}\right] .
\end{aligned}
$$

Moreover, we supplement the entropy condition

$$
U_{2} \cdot N_{X}<U_{\delta} \cdot N_{X}<U_{1} \cdot N_{X}
$$

where $N_{X}$ is the projection of the normal of the delta-shock on $X$-space (especially $X$-plane in two dimensions). The condition (2.14) means that all characteristics are incoming on two sides of the delta-shock defined in (2.10). With the notation (2.12), we rewrite (2.14) as

$$
\left[U_{1}, U_{\delta}, \frac{\partial X}{\partial s}\right]>0, \quad\left[U_{2}, U_{\delta}, \frac{\partial X}{\partial s}\right]<0 .
$$

Thus we get

$$
\frac{\partial w}{\partial t}=\rho_{1}\left[U_{1}, U_{\delta}, \frac{\partial X}{\partial s}\right]-\rho_{2}\left[U_{2}, U_{\delta}, \frac{\partial X}{\partial s}\right]>0 .
$$

This implies that the weight becomes heavier as the time passes, which reflects that particles stick more and more.

Remarks. (1). The relation (2.11) or (2.13) can be extended to the $n$-dimensional case $(n>3)$.

(2). The relation (2.11) is a system of the first-order partial differential equations with $(2 n+1)$-fold eigenvalue $\lambda=0$. It is somewhat similar to the linear system of hyperbolic partial differential equations.

Next we construct the solutions of (1.1) and (2.1) by two cases.

CASE 1. $[U] \cdot N>0$.

We solve (2.11) with the Cauchy data on $\Gamma$

$$
t=0: x_{1}=-\alpha_{2} s_{1}-\alpha_{3} s_{2}, \quad x_{2}=\alpha_{1} s_{1}, \quad x_{3}=\alpha_{1} s_{2}, \quad w=0,
$$

where $\alpha_{1}>0$ is assumed without losing the generality of $N$.

Multiplying the second equation of $(2.11)$ by $U_{2}$, then we subtract it from the third equation to yield

$$
\frac{\partial}{\partial t}\left(w\left(U_{\delta}-U_{2}\right)\right)=\rho_{1}\left(U_{1}-U_{2}\right)\left[U_{1}, U_{\delta}, \frac{\partial X}{\partial s}\right] .
$$

From this we have

$$
\begin{aligned}
& \left(u_{31}-u_{32}\right) \frac{\partial}{\partial t}\left(w\left(u_{1 \delta}-u_{12}\right)\right)=\left(u_{11}-u_{12}\right) \frac{\partial}{\partial t}\left(w\left(u_{3 \delta}-u_{32}\right)\right) \\
& \left(u_{31}-u_{32}\right) \frac{\partial}{\partial t}\left(w\left(u_{2 \delta}-u_{22}\right)\right)=\left(u_{21}-u_{22}\right) \frac{\partial}{\partial t}\left(w\left(u_{3 \delta}-u_{32}\right)\right) .
\end{aligned}
$$


Integrating these from 0 to $t$,

$$
\begin{aligned}
& w\left(\left(u_{31}-u_{32}\right)\left(u_{1 \delta}-u_{12}\right)-\left(u_{11}-u_{12}\right)\left(u_{3 \delta}-u_{32}\right)\right)=0 \\
& w\left(\left(u_{31}-u_{32}\right)\left(u_{2 \delta}-u_{22}\right)-\left(u_{21}-u_{22}\right)\left(u_{3 \delta}-u_{32}\right)\right)=0 .
\end{aligned}
$$

Since $w>0$, we get

$$
\begin{aligned}
& u_{1 \delta}=\frac{\left[u_{1}\right]}{\left[u_{3}\right]} u_{3 \delta}+\frac{u_{12} u_{31}-u_{11} u_{32}}{\left[u_{3}\right]}, \\
& u_{2 \delta}=\frac{\left[u_{2}\right]}{\left[u_{3}\right]} u_{3 \delta}+\frac{u_{22} u_{31}-u_{21} u_{32}}{\left[u_{3}\right]} .
\end{aligned}
$$

It follows from the first equation of (2.11) that

$$
\begin{aligned}
& {\left[u_{3}\right] \frac{\partial x_{1}}{\partial t}-\left[u_{1}\right] \frac{\partial x_{3}}{\partial t}=u_{12} u_{31}-u_{11} u_{32}} \\
& {\left[u_{3}\right] \frac{\partial x_{2}}{\partial t}-\left[u_{2}\right] \frac{\partial x_{3}}{\partial t}=u_{22} u_{31}-u_{21} u_{32}}
\end{aligned}
$$

The solutions of the above equations with (2.17) are

$$
\begin{aligned}
& {\left[u_{3}\right] x_{1}-\left[u_{1}\right] x_{3}=\left(u_{12} u_{31}-u_{11} u_{32}\right) t-\left[u_{3}\right]\left(\alpha_{2} s_{1}+\alpha_{3} s_{2}\right)-\alpha_{1}\left[u_{1}\right] s_{2},} \\
& {\left[u_{3}\right] x_{2}-\left[u_{2}\right] x_{3}=\left(u_{22} u_{31}-u_{21} u_{32}\right) t+\alpha_{1}\left[u_{3}\right] s_{1}-\alpha_{1}\left[u_{2}\right] s_{2} .}
\end{aligned}
$$

Differentiating (2.21) for $s_{1}$ and $s_{2}$ respectively, we obtain

$$
\begin{aligned}
& {\left[u_{3}\right] \frac{\partial x_{1}}{\partial s_{1}}-\left[u_{1}\right] \frac{\partial x_{3}}{\partial s_{1}}=-\alpha_{2}\left[u_{3}\right]} \\
& {\left[u_{3}\right] \frac{\partial x_{1}}{\partial s_{2}}-\left[u_{1}\right] \frac{\partial x_{3}}{\partial s_{2}}=-\alpha_{3}\left[u_{3}\right]-\alpha_{1}\left[u_{1}\right]} \\
& {\left[u_{3}\right] \frac{\partial x_{2}}{\partial s_{1}}-\left[u_{2}\right] \frac{\partial x_{3}}{\partial s_{1}}=\alpha_{1}\left[u_{3}\right]} \\
& {\left[u_{3}\right] \frac{\partial x_{2}}{\partial s_{2}}-\left[u_{2}\right] \frac{\partial x_{3}}{\partial s_{2}}=-\alpha_{1}\left[u_{2}\right]}
\end{aligned}
$$

These are

$$
\frac{\partial x_{1}}{\partial s_{1}}=\frac{\left[u_{1}\right]}{\left[u_{3}\right]} \frac{\partial x_{3}}{\partial s_{1}}-\alpha_{2}, \quad \frac{\partial x_{1}}{\partial s_{2}}=\frac{\left[u_{1}\right]}{\left[u_{3}\right]} \frac{\partial x_{3}}{\partial s_{2}}-\alpha_{1} \frac{\left[u_{1}\right]}{\left[u_{3}\right]}-\alpha_{3},
$$

and

$$
\frac{\partial x_{2}}{\partial s_{1}}=\frac{\left[u_{2}\right]}{\left[u_{3}\right]} \frac{\partial x_{3}}{\partial s_{1}}+\alpha_{1}, \quad \frac{\partial x_{2}}{\partial s_{2}}=\frac{\left[u_{2}\right]}{\left[u_{3}\right]} \frac{\partial x_{3}}{\partial s_{2}}-\alpha_{1} \frac{\left[u_{2}\right]}{\left[u_{3}\right]} .
$$

So, one can compute that

$$
\begin{aligned}
N_{x_{1}} & =\frac{\partial x_{2}}{\partial s_{1}} \frac{\partial x_{3}}{\partial s_{2}}-\frac{\partial x_{2}}{\partial s_{2}} \frac{\partial x_{3}}{\partial s_{1}}=\alpha_{1} \frac{\left[u_{2}\right]}{\left[u_{3}\right]} \frac{\partial x_{3}}{\partial s_{1}}+\alpha_{1} \frac{\partial x_{3}}{\partial s_{2}} \\
N_{x_{2}} & =-\left(\frac{\partial x_{1}}{\partial s_{1}} \frac{\partial x_{3}}{\partial s_{2}}-\frac{\partial x_{1}}{\partial s_{2}} \frac{\partial x_{3}}{\partial s_{1}}\right)=-\alpha_{1} \frac{\left[u_{1}\right]}{\left[u_{3}\right]} \frac{\partial x_{3}}{\partial s_{1}}-\alpha_{3} \frac{\partial x_{3}}{\partial s_{1}}+\alpha_{2} \frac{\partial x_{3}}{\partial s_{2}} \\
N_{x_{3}} & =\frac{\partial x_{1}}{\partial s_{1}} \frac{\partial x_{2}}{\partial s_{2}}-\frac{\partial x_{1}}{\partial s_{2}} \frac{\partial x_{2}}{\partial s_{1}} \\
& =\alpha_{3} \frac{\left[u_{2}\right]}{\left[u_{3}\right]} \frac{\partial x_{3}}{\partial s_{1}}-\frac{\alpha_{1}\left[u_{1}\right]+\alpha_{2}\left[u_{2}\right]}{\left[u_{3}\right]} \frac{\partial x_{3}}{\partial s_{2}}+\frac{\alpha_{1}([U] \cdot N)}{\left[u_{3}\right]}
\end{aligned}
$$


and

$$
\begin{aligned}
N_{t}= & -\left(u_{1 \delta} N_{x_{1}}+u_{2 \delta} N_{x_{2}}+u_{3 \delta} N_{x_{3}}\right) \\
= & \frac{\alpha_{1}\left(u_{11} u_{22}-u_{12} u_{21}\right)+\alpha_{3}\left(u_{31} u_{22}-u_{32} u_{21}\right)}{\left[u_{3}\right]} \frac{\partial x_{3}}{\partial s_{1}} \\
& +\frac{\alpha_{1}\left(u_{11} u_{32}-u_{12} u_{31}\right)+\alpha_{2}\left(u_{21} u_{32}-u_{22} u_{31}\right)}{\left[u_{3}\right]} \frac{\partial x_{3}}{\partial s_{2}} \\
& -\frac{\alpha_{1}([U] \cdot N)}{\left[u_{3}\right]} u_{3 \delta} .
\end{aligned}
$$

From (2.24)-(2.27), we arrive at

$$
\begin{aligned}
\frac{\partial w}{\partial t} & =[\rho] N_{t}+\left[\rho u_{1}\right] N_{x_{1}}+\left[\rho u_{2}\right] N_{x_{2}}+\left[\rho u_{3}\right] N_{x_{3}} \\
& =\frac{\alpha_{1}([U] \cdot N)}{\left[u_{3}\right]}\left(-[\rho] u_{3 \delta}+\left[\rho u_{3}\right]\right) .
\end{aligned}
$$

Noting that $\frac{\partial x_{3}}{\partial t}=u_{3 \delta}$, we get

$$
\frac{\partial}{\partial t}\left(w+\frac{\alpha_{1}([U] \cdot N)}{\left[u_{3}\right]}[\rho] x_{3}\right)=\frac{\alpha_{1}([U] \cdot N)}{\left[u_{3}\right]}\left[\rho u_{3}\right]
$$

which gives

$$
w+\frac{\alpha_{1}([U] \cdot N)}{\left[u_{3}\right]}[\rho] x_{3}=\frac{\alpha_{1}([U] \cdot N)}{\left[u_{3}\right]}\left(\left[\rho u_{3}\right] t+\alpha_{1}[\rho] s_{2}\right) .
$$

Similarly, one obtains

$$
\frac{\partial\left(w u_{3 \delta}\right)}{\partial t}=\frac{\alpha_{1}([U] \cdot N)}{\left[u_{3}\right]}\left(-\left[\rho u_{3}\right] u_{3 \delta}+\left[\rho u_{3}^{2}\right]\right),
$$

which gives

$$
w u_{3 \delta}+\frac{\alpha_{1}([U] \cdot N)}{\left[u_{3}\right]}\left[\rho u_{3}\right] x_{3}=\frac{\alpha_{1}([U] \cdot N)}{\left[u_{3}\right]}\left(\left[\rho u_{3}^{2}\right] t+\alpha_{1}\left[\rho u_{3}\right] s_{2}\right) .
$$

It follows from (2.29) and (2.30) that

$$
\left([\rho] u_{3 \delta}-\left[\rho u_{3}\right]\right) x_{3}=\left(\left[\rho u_{3}\right] t+\alpha_{1}[\rho] s_{2}\right) u_{3 \delta}-\left(\left[\rho u_{3}^{2}\right] t+\alpha_{1}\left[\rho u_{3}\right] s_{2}\right)
$$

because $\alpha_{1}([U] \cdot N) \neq 0$. We rewrite $(2.24)$ as

$$
\frac{\partial}{\partial t}\left(\frac{[\rho]}{2} x_{3}^{2}-\left(\left[\rho u_{3}\right] t+\alpha_{1}[\rho] s_{2}\right) x_{3}\right)=-\left(\left[\rho u_{3}^{2}\right] t+\alpha_{1}\left[\rho u_{3}\right] s_{2}\right),
$$

whose solution is

$$
\frac{[\rho]}{2} x_{3}^{2}-\left(\left[\rho u_{3}\right] t+\alpha_{1}[\rho] s_{2}\right) x_{3}+\frac{[\rho]}{2}\left(\alpha_{1} s_{2}\right)^{2}+\frac{\left[\rho u_{3}^{2}\right]}{2} t^{2}+\alpha_{1}\left[\rho u_{3}\right] s_{2} t=0
$$

when $\rho_{1} \neq \rho_{2}$. That is,

$$
x_{3}=\frac{1}{[\rho]}\left(\left[\rho u_{3}\right] \pm \sqrt{\rho_{1} \rho_{2}}\left[u_{3}\right]\right) t+\alpha_{1} s_{2} .
$$

Substituting (2.32) into (2.21), we get

$$
x_{2}=\frac{1}{[\rho]}\left(\left[\rho u_{2}\right] \pm \sqrt{\rho_{1} \rho_{2}}\left[u_{2}\right]\right) t+\alpha_{1} s_{1}
$$


and

$$
x_{1}=\frac{1}{[\rho]}\left(\left[\rho u_{1}\right] \pm \sqrt{\rho_{1} \rho_{2}}\left[u_{1}\right]\right) t-\alpha_{2} s_{1}-\alpha_{3} s_{2} .
$$

Therefore,

$$
U_{\delta}=\left(u_{1 \delta}, u_{2 \delta}, u_{3 \delta}\right)=\frac{\sqrt{\rho_{1}} U_{1} \pm \sqrt{\rho_{2}} U_{2}}{\sqrt{\rho_{1}} \pm \sqrt{\rho_{2}}} .
$$

By the entropy condition (2.14), we take

$$
U_{\delta}=\frac{\sqrt{\rho_{1}} U_{1}+\sqrt{\rho_{2}} U_{2}}{\sqrt{\rho_{1}}+\sqrt{\rho_{2}}}
$$

because the other does not satisfy (2.14). Thus we have

$$
X(t, s)=\left(x_{1}, x_{2}, x_{3}\right)(t, s)=\left(u_{1 \delta} t-\alpha_{2} s_{1}-\alpha_{3} s_{2}, u_{2 \delta} t+\alpha_{1} s_{1}, u_{3 \delta} t+\alpha_{1} s_{2}\right),
$$

where $U_{\delta}=\left(u_{1 \delta}, u_{2 \delta}, u_{3 \delta}\right)$ as in (2.35), and the speed is

$$
\sigma=U_{\delta} \cdot N=\frac{\left(\sqrt{\rho_{1}} U_{1}+\sqrt{\rho_{2}} U_{2}\right) \cdot N}{\sqrt{\rho_{1}}+\sqrt{\rho_{2}}} .
$$

From (2.28) or (2.29), we obtain

$$
w(t)=\frac{\alpha_{1}([U] \cdot N)}{\left[u_{3}\right]}\left(-[\rho] u_{3 \delta}+\left[\rho u_{3}\right]\right) t=\alpha_{1} \sqrt{\rho_{1} \rho_{2}}([U] \cdot N) t .
$$

When $\rho_{1}=\rho_{2}$, one can easily solve $U_{\delta}=\frac{U_{1}+U_{2}}{2}$, etc.

Thus we obtain the delta-shock $X(t, s)=U_{\delta} t+X(0, s)$, where $X(0, s)=\left(-\alpha_{2} s_{1}-\right.$ $\left.\alpha_{3} s_{2}, \alpha_{1} s_{1}, \alpha_{1} s_{2}\right)$ is given in (2.10), which separated $(t, X)$-space into two parts $\Omega_{1}$ and $\Omega_{2}$. In $\Omega_{1}$ the solution is the constant state $\left(\rho_{1}, U_{1}\right)$, while in $\Omega_{2}$ the solution is $\left(\rho_{2}, U_{2}\right)$. After eliminating the parametric variable $s$, the delta-shock solution can be formulated explicitly as in (1.3-1.6) with $w(t)$ in (2.31). This formula shows that delta-shock is a class of planar wave.

For the $n$-dimensional case $(n>3)$, the same procedure as above yields the delta-shock solution defined in (1.3-1.6) with

$$
w(t)=\alpha_{1}^{n-2} \sqrt{\rho_{1} \rho_{2}}([U] \cdot N) t
$$

which holds for $n=2$ and $n=3$ too.

The above results show that the delta-shock solution depends exactly on a family of one parameter. Therefore, to guarantee the uniqueness of the delta-shock, we choose

$$
w_{\delta}(t)=\frac{1}{\alpha_{1}^{n-2}} w(t) \text { when }|N|=1
$$

and

$$
w_{\delta}(t)=\frac{1}{\alpha_{1}^{n-2}|N|} w(t) \text { when }|N| \neq 1
$$

with the physical consideration and the following viscosity vanishing process.

CASE 2. $[U] \cdot N<0$. 
This case is simple. For the completeness, we construct the solution as

$$
(\rho, U)(t, X)= \begin{cases}\left(\rho_{1}, U_{1}\right), & X \cdot N<\left(U_{1} \cdot N\right) t \\ \operatorname{Vac}, & \left(U_{1} \cdot N\right) t \leq X \cdot N \leq\left(U_{2} \cdot N\right) t \\ \left(\rho_{2}, U_{2}\right), & X \cdot N>\left(U_{2} \cdot N\right) t\end{cases}
$$

where Vac denotes the vacuum solution (see Sec. 4).

REMARK. For the general case, the structure of solutions is just the combination of solutions of Case 1 and Case 2 besides the contact discontinuity.

3. Existence of solutions for the viscous system (1.8). In this section we consider the existence of solutions for the Riemann problem (1.8) and (2.1) in $n(n \geq 2)$ dimensions. Performing the self-similar transformation $\xi=\frac{X \cdot N}{t}$, we get the system of ordinary differential equations

$$
\left\{\begin{array}{l}
-\xi \rho_{\xi}+(\rho(U \cdot N))_{\xi}=0, \\
-\xi(\rho U)_{\xi}+(\rho U(U \cdot N))_{\xi}=\varepsilon U_{\xi \xi} .
\end{array}\right.
$$

The initial condition becomes the boundary condition

$$
(\rho, U)(-\infty)=\left(\rho_{1}, U_{1}\right), \quad(\rho, U)(+\infty)=\left(\rho_{2}, U_{2}\right)
$$

To solve the two-point boundary value problem (3.1-3.3) of high-order ordinary differential equations with the boundary value at infinity, we first consider the existence of solutions of the system $(3.1-3.2)$ in the interval $[-R, R]$, where $R$ is a sufficiently large real number, with the boundary value

$$
\left\{\begin{array}{l}
(\rho, U)(-R)=\left(\rho_{1}, U_{1}\right), \\
(\rho, U)(+R)=\left(\rho_{2}, U_{2}\right) .
\end{array}\right.
$$

This is the content of the following theorem.

THEOREM 3.1. There exists a weak solution

$$
(\rho, U) \in L^{1}[-R, R] \times\left(C^{2}[-R, R]\right)^{n}
$$

for the boundary value problem (3.1-3.2) and (3.4).

The theorem is obtained by Schauder's fixed point theorem and follows from the next three lemmas.

Take

$$
\begin{aligned}
& B=\left(C^{2}[-R, R]\right)^{n} \\
& K=\left\{V \mid V \in B, V(-R)=U_{1}, V(+R)=U_{2}, V \text { and } V \cdot N \text { are monotone }\right\} .
\end{aligned}
$$

Obviously, $K$ is a convex closed set in $B$, a Banach space.

Lemma 3.2. For any $V \in K$, the problem

$$
\left\{\begin{array}{l}
-\xi \rho_{\xi}+(\rho(V \cdot N))_{\xi}=0, \\
\rho(-R)=\rho_{1}, \rho(+R)=\rho_{2},
\end{array}\right.
$$

possesses a weak solution $\rho \in L^{1}[-R, R]$. 
(i). When $[U] \cdot N>0$,

$$
\rho(\xi)= \begin{cases}\rho_{-}(\xi), & -R \leq \xi<\xi_{\sigma}, \\ \rho_{+}(\xi), & \xi_{\sigma}<\xi \leq+R,\end{cases}
$$

where $\xi_{\sigma}$ is a unique solution of equation

$$
V\left(\xi_{\sigma}\right) \cdot N=\xi_{\sigma},
$$

$\rho_{-}(\xi)$ is monotone increasing in $\left(-R, \xi_{\sigma}\right)$, while $\rho_{+}(\xi)$ is monotone decreasing in $\left(\xi_{\sigma},+R\right)$.

(ii). When $[U] \cdot N<0$,

$$
\rho(\xi)= \begin{cases}\rho_{-}(\xi), & -R \leq \xi<\xi_{\sigma_{1}} \\ 0, & \xi_{\sigma_{1}} \leq \xi \leq \xi_{\sigma_{2}} \\ \rho_{+}(\xi), & \xi_{\sigma_{2}}<\xi \leq+R\end{cases}
$$

where $\xi_{\sigma_{1}} \leq \xi_{\sigma_{2}}$ satisfying

$$
\xi_{\sigma_{1}}=\min \{\xi \mid V(\xi) \cdot N=\xi\}, \quad \xi_{\sigma_{2}}=\max \{\xi \mid V(\xi) \cdot N=\xi\}
$$

and

$$
\lim _{\xi \rightarrow \xi_{\sigma_{1}}^{-}} \rho_{-}(\xi)=\lim _{\xi \rightarrow \xi_{\sigma_{2}}^{+}} \rho_{+}(\xi)=0,
$$

$\rho_{-}(\xi)$ is monotone decreasing in $\left(-R, \xi_{\sigma_{1}}\right)$, while $\rho_{+}(\xi)$ is monotone increasing in $\left(\xi_{\sigma_{2}},+R\right)$.

In particular, $\rho_{-}(\xi)$ and $\rho_{+}(\xi)$ in (3.7) and (3.9) can be formulated as

$$
\rho_{-}(\xi)=\rho_{1} \exp \left(\int_{-R}^{\xi} \frac{-V^{\prime}(s) \cdot N}{V(s) \cdot N-s} d s\right)
$$

and

$$
\rho_{+}(\xi)=\rho_{2} \exp \left(\int_{\xi}^{R} \frac{V^{\prime}(s) \cdot N}{V(s) \cdot N-s} d s\right)
$$

where $\prime=\frac{d}{d \xi}$.

Proof. The problem (3.5-3.6) is a two-point boundary value problem for the first-order degenerate ordinary equation. The singularity point of (3.5) is given by the solution of equation (3.8).

(i). When $[U] \cdot N>0, V(\xi) \cdot N$ is decreasing; so the singularity point is unique, denoted by $\xi_{\sigma}$. Integrating the equation (3.5) from $\rho(-R)=\rho_{1}$ or $\rho(+R)=\rho_{2}$ respectively, we obtain the solution (3.7) with (3.12-3.13), which immediately shows that $\rho_{-}(\xi)$ and $\rho_{+}(\xi)$ are monotone for $\xi<\xi_{\sigma}$ respectively. Furthermore, one easily obtains

$$
\lim _{\xi \rightarrow \xi_{\sigma}^{-}} \rho_{-}(\xi)=+\infty, \quad \lim _{\xi \rightarrow \xi_{\sigma}^{+}} \rho_{+}(\xi)=+\infty .
$$


We proceed to prove that $\rho(\xi)$ is a weak solution of $(3.5)$ and $\rho \in L^{1}[-R,+R]$. Integrating (3.5) on $[-R, \xi]$ for $-R<\xi<\xi_{\sigma}$, we get

$$
(V(\xi) \cdot N-\xi) \rho_{-}(\xi)-\left(U_{1} \cdot N+R\right) \rho_{1}+\int_{-R}^{\xi} \rho_{-}(r) d r=0
$$

Set

$$
\rho(\xi)=\int_{-R}^{\xi} \rho_{-}(r) d r, \quad A_{1}=\left(U_{1} \cdot N+R\right) \rho_{1}, \quad a(\xi)=V(\xi) \cdot N-\xi
$$

Then the equation (3.14) can be written as

$$
\left\{\begin{array}{l}
a(\xi) \rho^{\prime}(\xi)+p(\xi)=A_{1} \\
p(-R)=0
\end{array}\right.
$$

It follows that

$$
p(\xi)=A_{1}\left\{1-\exp \left(-\int_{-R}^{\xi} \frac{d r}{a(r)}\right)\right\} .
$$

Noting that $a(\xi)>0$ and $a(\xi)=O\left(\left|\xi-\xi_{\sigma}\right|\right)$ as $\xi \rightarrow \xi_{\sigma}^{-}$, we obtain

$$
\lim _{\xi \rightarrow \xi_{\sigma}^{-}} \int_{-R}^{\xi} \rho_{-}(r) d r=A_{1}
$$

Hence

$$
\lim _{\xi \rightarrow \xi_{\sigma}^{-}}(V(\xi) \cdot N-\xi) \rho_{-}(\xi)=0
$$

Similarly, one can get

$$
\begin{gathered}
\lim _{\xi \rightarrow \xi_{\sigma}^{+}} \int_{R}^{\xi} \rho_{+}(r) d r=A_{2}, \\
\lim _{\xi \rightarrow \xi_{\sigma}^{+}}(V(\xi) \cdot N-\xi) \rho_{+}(\xi)=0,
\end{gathered}
$$

where $A_{2}=\left(U_{2} \cdot N-R\right) \rho_{2}$. The equalities (3.16) and (3.18) imply that $\rho(\xi) \in L^{1}[-R, R]$.

For arbitrary $\phi \in C_{0}^{\infty}[-R, R]$, we verify that

$$
-\int_{-R}^{R}(V \cdot N-\xi) \rho \phi^{\prime} d \xi+\int_{-R}^{R} \rho \phi d \xi=0 .
$$

Indeed, for any $\xi_{1}, \xi_{2}$, such that $-R<\xi_{1}<\xi_{\sigma}<\xi_{2}<R$,

$$
\begin{aligned}
I & =-\int_{-R}^{R}(V \cdot N-\xi) \rho \phi^{\prime} d \xi+\int_{-R}^{R} \rho \phi d \xi \\
& =\left(\int_{-R}^{\xi_{1}}+\int_{-\xi_{1}}^{\xi_{2}}+\int_{\xi_{2}}^{R}\right)\left(-(V \cdot N-\xi) \rho \phi^{\prime}+\rho \phi\right) d \xi \\
& =I_{1}+I_{2}+I_{3} .
\end{aligned}
$$


By (3.17) and (3.19),

$$
\begin{aligned}
\left|I_{1}\right| & =\left|-\left(V\left(\xi_{1}\right) \cdot N-\xi_{1}\right) \rho_{-}\left(\xi_{1}\right) \phi\left(\xi_{1}\right)+\int_{-R}^{\xi_{1}}\left(\left((V \cdot N-\xi) \rho^{\prime}\right) \phi+\rho \phi\right) d \xi\right| \\
& =\left|-\left(V\left(\xi_{1}\right) \cdot N-\xi_{1}\right) \rho_{-}\left(\xi_{1}\right) \phi\left(\xi_{1}\right)\right| \rightarrow 0, \quad \text { as } \xi_{1} \rightarrow \xi_{\sigma}^{-}, \\
\left|I_{3}\right| & =\left|\left(V\left(\xi_{2}\right) \cdot N-\xi_{2}\right) \rho_{+}\left(\xi_{2}\right) \phi\left(\xi_{2}\right)\right| \rightarrow 0, \quad \text { as } \xi_{2} \rightarrow \xi_{\sigma}^{+},
\end{aligned}
$$

and since $\rho \in L^{1}[-R, R]$,

$$
\left|I_{2}\right| \leq \int_{\xi_{1}}^{\xi_{2}}\left|-(V \cdot N-\xi) \phi^{\prime}+\phi\right||\rho| d \xi \rightarrow 0, \quad \text { as } \xi_{1} \rightarrow \xi_{\sigma}^{-}, \xi_{2} \rightarrow \xi_{\sigma}^{+} .
$$

$I$ is independent of $\xi_{1}$ and $\xi_{2}$; so $I=0$, i.e., (3.20) holds. Therefore, $\rho(\xi)$ defined in (3.7) is a weak solution of (3.5).

(ii). When $[U] \cdot N<0, V(\xi) \cdot N$ is increasing; so we get $\xi_{\sigma_{1}} \leq \xi_{\sigma_{2}}$. Thus we can find the following $\rho(\xi)$ of (3.5) to be (3.9) with (3.12-3.13). Because

$$
\begin{aligned}
\int_{-R}^{\xi} \frac{V^{\prime}(s) \cdot N}{V(s) \cdot N-s} d s & =\left(V^{\prime}(\zeta) \cdot N\right) \int_{-R}^{\xi} \frac{d s}{V(s) \cdot N-s} \\
& \geq\left(V^{\prime}(\zeta) \cdot N\right) \int_{-R}^{\xi} \frac{d s}{V(\xi) \cdot N-s} \\
& =-\left(V^{\prime}(\zeta) \cdot N\right) \ln \frac{V(\xi) \cdot N-\xi}{V(\xi) \cdot N+R} \\
& \rightarrow+\infty, \quad \text { as } \xi \rightarrow \xi_{\sigma_{1}}^{-},
\end{aligned}
$$

where $-R<\xi<\xi_{\sigma_{1}},-R \leq \zeta \leq \xi$, we get the first half of (3.11). The second half can be obtained in a similar way. In addition, the monotonicity of $\rho_{-}(\xi)$ and $\rho_{+}(\xi)$ is obvious. The proof of Lemma 3.2 is completed.

Define an operator $T: K \rightarrow B$ as follows: for any $V \in K, U=T V$ is the unique solution of the following boundary value problem:

$$
\left\{\begin{array}{l}
\varepsilon U^{\prime \prime}=\rho(V, \xi)(V \cdot N-\xi) U^{\prime} \\
U(-R)=U_{1}, \quad U(+R)=U_{2}
\end{array}\right.
$$

where $\rho(V, \xi)$ is defined in (3.7) or (3.9). In fact, the solution of this problem can be found to be

$$
U(\xi)=\frac{\left(U_{2}-U_{1}\right) \int_{-R}^{\xi} \exp \left(\int_{-R}^{r} \frac{\rho(V \cdot N-s)}{\varepsilon} d s\right) d r}{\int_{-R}^{R} \exp \left(\int_{-R}^{r} \frac{\rho(V \cdot N-s)}{\varepsilon} d s\right) d r}+U_{1} .
$$

Lemma 3.3. $T: K \rightarrow K$ is a continuous operator in $B$.

Proof. Take $V_{m} \rightarrow V$ (in $\left.B\right)(m \rightarrow \infty), V_{m}, V \in K$. Then

$$
U_{m}=T V_{m}, \quad U=T V
$$


satisfy (3.21) and (3.22), and we have

$$
\left\{\begin{aligned}
\varepsilon\left(U_{m}-U\right)^{\prime \prime}= & \rho_{m}\left(V_{m} \cdot N-\xi\right)\left(U_{m}-U\right)^{\prime} \\
& +\left(\rho_{m}\left(V_{m} \cdot N-\xi\right)-\rho(V \cdot N-\xi)\right) U^{\prime} \\
\left(U_{m}-U\right)( \pm R) & =O .
\end{aligned}\right.
$$

It can be solved to give

$$
\begin{aligned}
\left(U_{m}-U\right)^{\prime}(\xi)= & -\frac{\int_{-R}^{R} \int_{-R}^{t} Q_{m}(r) \exp \left(\int_{r}^{t} p_{m}(s) d s\right) d r d t}{\int_{-R}^{R} \exp \left(\int_{-R}^{r} p_{m}(s) d s\right) d r} \exp \left(\int_{-R}^{\xi} p_{m}(s) d s\right) \\
& +\int_{-R}^{\xi} Q_{m}(r) \exp \left(\int_{r}^{\xi} p_{m}(s) d s\right) d r \\
\left(U_{m}-U\right)(\xi)= & -\frac{\int_{-R}^{R} \int_{-R}^{t} Q_{m}(r) \exp \left(\int_{r}^{t} p_{m}(s) d s\right) d r d t}{\int_{-R}^{R} \exp \left(\int_{-R}^{r} p_{m}(s) d s\right) d r} \int_{-R}^{\xi} \exp \left(\int_{-R}^{r} p_{m}(s) d s\right) d r \\
& +\int_{-R}^{\xi} \int_{-R}^{t} Q_{m}(r) \exp \left(\int_{r}^{t} p_{m}(s) d s\right) d r d t
\end{aligned}
$$

where $p_{m}=\rho_{m}\left(V_{m} \cdot N-\xi\right), Q_{m}=\left(\rho_{m}\left(V_{m} \cdot N-\xi\right)-\rho(V \cdot N-\xi)\right) U^{\prime}$. From the equation (3.5), we have

$$
(\rho(V \cdot N-\xi))^{\prime}=-\rho<0, \quad\left(\rho_{m}\left(V_{m} \cdot N-\xi\right)\right)^{\prime}=-\rho_{m}<0 \quad(m=1,2, \ldots) .
$$

Then $\rho(V \cdot N-\xi)$ and $\rho_{m}\left(V_{m} \cdot N-\xi\right)(m=1,2, \ldots)$ are monotone decreasing and continuous functions. Because the sequence of monotone functions (continuous or discontinuous) which converges to a continuous function must converge uniformly, we get that $Q_{m}(\xi)$ converges to $O$ uniformly. Thus it follows from (3.24) and (3.26-3.27) that

$$
U_{m} \rightarrow U(\text { in } B) \quad \text { as } m \rightarrow \infty \text {. }
$$

Therefore $T: K \rightarrow B$ is a continuous operator in $B$.

Moreover, from the equations (3.21), we have

$$
U^{\prime}(\xi)=U^{\prime}(0) \exp \left(\int_{0}^{\xi} \frac{\rho(V \cdot N-s)}{\varepsilon} d s\right)
$$

and

$$
U^{\prime}(\xi) \cdot N=\left(U^{\prime}(0) \cdot N\right) \exp \left(\int_{0}^{\xi} \frac{\rho(V \cdot N-s)}{\varepsilon} d s\right),
$$

which imply that $U=T V$ and $U \cdot N=T(V \cdot N)$ are all monotone. This result can also be obtained from (3.23). Thus, we get $T K \subset K$. The proof of the lemma is completed.

Lemma 3.4. $T K$ is precompact in $B$. 
Proof. According to the continuity of $T$ and the Ascoli-Arzela theorem [A], it remains to show the boundedness of $T K$ in $B$.

When $[U] \cdot N>0$, for any $V \in K$, we have

$$
U^{\prime}(\xi)=U^{\prime}(-R) \exp \left(\int_{-R}^{\xi} \frac{\rho(V \cdot N-s)}{\varepsilon} d s\right) .
$$

By Lemma 3.2 and when $s<\xi_{\sigma}$,

$$
0<\rho(V \cdot N-\xi)=\rho_{1}\left(U_{1} \cdot N+R\right)-\int_{-R}^{s} \rho d r<\rho_{1}\left(U_{1} \cdot N+R\right) .
$$

When $s>\xi_{\sigma}$,

$$
0>\rho(V \cdot N-s)=\rho_{2}\left(U_{2} \cdot N-R\right)+\int_{s}^{R} \rho d r>\rho_{2}\left(U_{2} \cdot N-R\right) .
$$

Thus we need to consider only the uniform boundedness of $U^{\prime}(-R)$. From the equation (3.21), one can deduce that

$$
u_{i}^{\prime \prime}(\xi)<0 \quad \text { if } u_{i 1}>u_{i 2}
$$

and

$$
u_{i}^{\prime \prime}(\xi)>0 \quad \text { if } u_{i 1}<u_{i 2},
$$

where $\xi \in\left[-R, \xi_{\sigma}\right), i=1,2, \ldots, n$. Then in $\left[-R, \xi_{\sigma}\right)$, we have

$$
u_{i}^{\prime}(\xi)<u_{i}^{\prime}(-R)<0 \quad \text { if } u_{i 1}>u_{i 2}
$$

and

$$
u_{i}^{\prime}(\xi)>u_{i}^{\prime}(-R)>0 \quad \text { if } u_{i 1}<u_{i 2}
$$

If $u_{i 1}>u_{i 2}$, then

$$
\begin{aligned}
u_{i 1}-u_{i 2}>u_{i}(-R)-u_{i}\left(\xi_{\sigma}\right) & =u_{i}^{\prime}(\zeta)\left(-R-\xi_{\sigma}\right) \\
& >u_{i}^{\prime}(\zeta)\left(-R-U_{2} \cdot N\right), \quad \zeta \in\left(-R, \xi_{\sigma}\right) .
\end{aligned}
$$

So

$$
0>u_{i}^{\prime}(-R)>u_{i}^{\prime}(\zeta)>-\frac{u_{i 1}-u_{i 2}}{R+U_{2} \cdot N}, \quad i=1,2, \ldots, n .
$$

Analogously, if $u_{i 1}<u_{i 2}$, we also get

$$
0<u_{i}^{\prime}(-R)<-\frac{u_{i 1}-u_{i 2}}{R+U_{2} \cdot N}, \quad i=1,2, \ldots, n .
$$

These above imply that $U^{\prime}(\xi)$ is uniformly bounded for $K$.

When $[U] \cdot N<0$, we can obtain the same result in a similar way. Therefore, $U(\xi), U^{\prime}(\xi)$ and $U^{\prime \prime}(\xi)$ are all uniformly bounded for $K$, that is, $T K$ is a bounded set in $B$.

In what follows, we extend the solutions of $(3.1-3.2)$ in $[-R, R]$ to the whole interval $(-\infty, \infty)$. To this end, we need the following lemma.

LEmma 3.5. The solution $(\rho, U)(\xi)$ of the system (3.1-3.2) with boundary value (3.4) satisfies

(i). $U(\xi)$ and $U^{\prime}(\xi)$ have uniform bounds independent of $R$; 
(ii). $\left|U^{\prime \prime}(\xi)\right| \leq C(\varepsilon), \xi \in[-R, R]$, where $C(\varepsilon)$ is a constant only dependent on $\varepsilon$;

(iii). $\rho_{R}(\xi)$ converges as $R \rightarrow+\infty$.

Proof. We only prove the case $[U] \cdot N>0$; for the case $[U] \cdot N<0$, one can verify it similarly. At this moment, we have

$$
U_{2} \cdot N<\xi_{\sigma}<U_{1} \cdot N
$$

(i). Take $-R<\xi_{1}<U_{2} \cdot N$. From (3.2) it follows that

$$
U^{\prime}(\xi)=U^{\prime}\left(\xi_{1}\right) \exp \left(\int_{\xi_{1}}^{\xi} \frac{\rho(U \cdot N-s)}{\varepsilon} d s\right) .
$$

For $\xi \in\left(-R, \xi_{\sigma}\right)$, and if $u_{i 1}>u_{i 2}$, then $u_{i}^{\prime \prime}(\xi)<0$; so $0>u_{i}^{\prime}\left(\xi_{1}\right)>u_{i}^{\prime}(\xi), \xi \in\left(\xi_{1}, \xi_{\sigma}\right)$, $i=1,2, \ldots, n$. Since

$$
u_{i 1}-u_{i 2}>u_{i}\left(\xi_{1}\right)-u_{i}\left(\xi_{\sigma}\right)=u_{i}^{\prime}(\zeta)\left(\xi_{1}-\xi_{\sigma}\right)>u_{i}^{\prime}(\zeta)\left(\xi_{1}-U_{2} \cdot N\right),
$$

where $\zeta \in\left(\xi_{1}, \xi_{\sigma}\right)$, we get

$$
u_{i}^{\prime}(\zeta)>\frac{u_{i 1}-u_{i 2}}{\xi_{1}-U_{2} \cdot N}, \quad \zeta \in\left(\xi_{1}, \xi_{\sigma}\right)
$$

It follows that

$$
0>u_{i}^{\prime}\left(\xi_{1}\right)>\frac{u_{i 1}-u_{i 2}}{\xi_{1}-U_{2} \cdot N}, \quad i=1,2, \ldots, n .
$$

Analogously, if $u_{i 1}<u_{i 2}$, then we also get

$$
0<u_{i}^{\prime}\left(\xi_{1}\right)<\frac{u_{i 1}-u_{i 2}}{\xi_{1}-U_{2} \cdot N}, \quad i=1,2, \ldots, n
$$

Thus, $U^{\prime}\left(\xi_{1}\right)=\left(u_{1}^{\prime}\left(\xi_{1}\right), u_{2}^{\prime}\left(\xi_{1}\right), \ldots, u_{n}^{\prime}\left(\xi_{1}\right)\right)$ is uniformly bounded independent of $R$. In addition,

$$
\begin{aligned}
\rho_{-}\left(\xi_{1}\right) & =\rho_{1} \exp \left(\int_{-R}^{\xi_{1}} \frac{-U^{\prime}(s) \cdot N}{U(s) \cdot N-s} d s\right) \\
& =\rho_{1} \exp \left(\int_{-R}^{\xi_{1}} \frac{-(U(s) \cdot N-s)^{\prime}-1}{U(s) \cdot N-s} d s\right) \\
& =\rho_{1} \frac{U_{1} \cdot N+R}{U\left(\xi_{1}\right) \cdot N-\xi_{1}} \exp \int_{-R}^{\xi_{1}} \frac{-d s}{U(s) \cdot N-s} d s \\
& \leq \rho_{1} \frac{U_{1} \cdot N+R}{U\left(\xi_{1}\right) \cdot N-\xi_{1}} \exp \int_{-R}^{\xi_{1}} \frac{-d s}{U_{1} \cdot N-s} d s \\
& =\frac{\rho_{1}\left(U_{1} \cdot N-\xi_{1}\right)}{U\left(\xi_{1}\right) \cdot N-\xi_{1}} .
\end{aligned}
$$

When $\xi<\xi_{1}$,

$$
\exp \int_{\xi_{1}}^{\xi} \frac{\rho(U \cdot N-s)}{\varepsilon} d s<1
$$


When $\xi_{1}<\xi<\xi_{\sigma}$, note that

$$
\begin{aligned}
\rho(U \cdot N-\xi) & =\rho\left(\xi_{1}\right)\left(U\left(\xi_{1}\right) \cdot N-\xi_{1}\right)-\int_{\xi_{1}}^{\xi} \rho d r \\
& \leq \rho\left(\xi_{1}\right)\left(U\left(\xi_{1}\right) \cdot N-\xi_{1}\right) \leq \rho_{1}\left(U_{1} \cdot N-\xi_{1}\right),
\end{aligned}
$$

we obtain

$$
\exp \int_{\xi_{1}}^{\xi} \frac{\rho(U \cdot N-s)}{\varepsilon} d s \leq \exp \frac{\rho_{1}\left(U_{1} \cdot N-\xi_{1}\right)^{2}}{\varepsilon}
$$

When $\xi>\xi_{\sigma}$,

$$
\begin{aligned}
\int_{\xi_{1}}^{\xi} \frac{\rho(U \cdot N-s)}{\varepsilon} d s & =\int_{\xi_{1}}^{\xi_{\sigma}} \frac{\rho(U \cdot N-s)}{\varepsilon} d s+\int_{\xi_{\sigma}}^{\xi} \frac{\rho(U \cdot N-s)}{\varepsilon} d s \\
& <\int_{\xi_{1}}^{\xi_{\sigma}} \frac{\rho(U \cdot N-s)}{\varepsilon} d s .
\end{aligned}
$$

Therefore, $U^{\prime}(\xi)$ and $U(\xi)$ have uniform bounds independent of $R$.

(ii). From the equation (3.2) and (i), we can easily get this result.

(iii). Completely similar to the estimate on $\rho\left(\xi_{1}\right)$ in (i), we can obtain that

$$
\rho_{-}(\xi) \leq \frac{\rho_{1}\left(U_{1} \cdot N-\xi\right)}{U(\xi) \cdot N-\xi}, \quad \xi \in\left(-R, \xi_{\sigma}\right)
$$

and

$$
\rho_{+}(\xi) \leq \frac{\rho_{2}\left(U_{2} \cdot N-\xi\right)}{U(\xi) \cdot N-\xi}, \quad \xi \in\left(\xi_{\sigma}, R\right)
$$

which shows that $\rho_{R}(U, \xi)$ converges as $R \rightarrow+\infty$.

By Lemma 3.5, for any $L>0,\left\{U_{R}(\xi)\right\}$ is a compact set in $\left(C^{1}[-L, L]\right)^{n}$ if $R>L$. Hence there exists a subsequence $\left\{U_{R_{i}}(\xi)\right\}$ such that

$$
\lim _{R_{i} \rightarrow+\infty} U_{R_{i}}(\xi)=U(\xi), \quad \lim _{R_{i} \rightarrow+\infty} U_{R_{i}}^{\prime}(\xi)=U^{\prime}(\xi), \quad \xi \in[-L, L] .
$$

Using the Helley selecting principle, we get a subsequence, also denoted by $\left\{U_{R_{i}}(\xi)\right\}$ such that

$$
\lim _{R_{i} \rightarrow+\infty} U_{R_{i}}(\xi)=U(\xi), \quad \lim _{R_{i} \rightarrow+\infty} U_{R_{i}}^{\prime}(\xi)=U^{\prime}(\xi), \quad \xi \in(-\infty,+\infty) .
$$

TheOREM 3.6. Let $\varepsilon \leq \varepsilon_{0}$. Then $U(\xi)$ satisfies

$$
\left\{\begin{array}{l}
\varepsilon U^{\prime \prime}=\rho(U, \xi)(U \cdot N-\xi) U^{\prime} \\
U(-\infty)=U_{1}, U(+\infty)=U_{2}
\end{array}\right.
$$

and

$$
\rho(\xi)= \begin{cases}\rho_{-}(\xi), & -\infty<\xi<\xi_{\sigma} \\ \rho_{+}(\xi), & \xi_{\sigma}<\xi<+\infty\end{cases}
$$


when $[U] \cdot N>0$, while

$$
\rho(\xi)= \begin{cases}\rho_{-}(\xi), & -\infty<\xi<\xi_{\sigma_{1}} \\ 0, & \xi_{\sigma_{1}} \leq \xi \leq \xi_{\sigma_{2}} \\ \rho_{+}(\xi), & \xi_{\sigma_{2}}<\xi<+\infty\end{cases}
$$

when $[U] \cdot N<0$, where

$$
\left\{\begin{array}{l}
\rho_{-}(\xi)=\rho_{1} \exp \left(\int_{-\infty}^{\xi} \frac{-U^{\prime}(s) \cdot N}{U(s) \cdot N-s} d s\right) \\
\rho_{+}(\xi)=\rho_{2} \exp \left(\int_{\xi}^{+\infty} \frac{U^{\prime}(s) \cdot N}{U(s) \cdot N-s} d s\right)
\end{array}\right.
$$

and $\xi_{\sigma}, \xi_{\sigma_{1}}$ and $\xi_{\sigma_{2}}$ satisfy

$$
\xi_{\sigma_{1}}=\min \left\{U\left(\xi_{\sigma}\right) \cdot N=\xi_{\sigma}\right\}, \quad \xi_{\sigma_{2}}=\max \left\{U\left(\xi_{\sigma}\right) \cdot N=\xi_{\sigma}\right\} .
$$

Proof. Denote by $\left(\rho_{R}, U_{R}\right)(\xi)$ the solution of (3.1-3.2) and (3.4). When $[U] \cdot N>0$, integrating (3.21) from $\xi_{2}$ to $\xi, \xi_{2}$ is a fixed point, then from the above lemmas we always have

$$
\begin{aligned}
\varepsilon\left(U_{R}^{\prime}(\xi)-U_{R}^{\prime}\left(\xi_{2}\right)\right)=\rho_{R}(\xi)\left(U_{R}(\xi)\right. & \cdot N-\xi) U_{R}(\xi) \\
& -\rho_{R}\left(\xi_{2}\right)\left(U_{R}\left(\xi_{2}\right) \cdot N-\xi_{2}\right) U_{R}\left(\xi_{2}\right)+\int_{\xi_{2}}^{\xi} \rho_{R} U_{R} d r
\end{aligned}
$$

whenever $\xi_{\sigma}$ is between $\xi_{2}$ and $\xi$ or not. Letting $R \rightarrow+\infty$, by the Lebesgue Convergence Theorem it follows that

$$
\varepsilon\left(U^{\prime}(\xi)-U^{\prime}\left(\xi_{2}\right)\right)=\rho(\xi)(U(\xi) \cdot N-\xi) U(\xi)-\rho\left(\xi_{2}\right)\left(U\left(\xi_{2}\right) \cdot N-\xi_{2}\right) U\left(\xi_{2}\right)+\int_{\xi_{2}}^{\xi} \rho U d r
$$

When $[U] \cdot N<0$, we can obtain (3.32) easily. Because the right side of (3.32) is continuous, we get

$$
U^{\prime} \in\left(C^{1}(-\infty,+\infty)\right)^{n}
$$

Differentiating (3.32) yields

$$
\varepsilon U^{\prime \prime}=\rho(U \cdot N-\xi) U^{\prime}
$$

and from (3.23) we have

$$
U(-\infty)=U_{1}, \quad U(+\infty)=U_{2}
$$

The formula of $\rho(\xi)$ in (3.29-3.31) can be obtained from the above lemmas. This completes the proof. 
4. The limit solutions of (3.1-3.3) as $\varepsilon \rightarrow 0^{+}$. This section concerns the behavior of solutions of (1.8) and (2.1) or (3.1-3.3) as $\varepsilon \rightarrow 0^{+}$and establishes the stability for delta-shocks in multidimensions.

CASE 1. $[U] \cdot N>0$.

LEMMA 4.1. Let $\xi_{\sigma}^{\varepsilon}$ be the unique point satisfying

$$
\xi_{\sigma}^{\varepsilon}=U^{\varepsilon}\left(\xi_{\sigma}^{\varepsilon}\right) \cdot N, \quad \xi_{\sigma}=\lim _{\varepsilon \rightarrow 0^{+}} \xi_{\sigma}^{\varepsilon}
$$

(pass to a subsequence if necessary). Then for any $\eta>0$,

$$
\begin{aligned}
& \lim _{\varepsilon \rightarrow 0^{+}} U_{\xi}^{\varepsilon}(\xi)=O \quad \text { for }\left|\xi-\xi_{\sigma}\right| \geq \eta, \\
& \lim _{\varepsilon \rightarrow 0^{+}} U_{\xi}^{\varepsilon}(\xi)= \begin{cases}U_{1} & \text { for } \xi \leq \xi_{\sigma}-\eta \\
U_{2} & \text { for } \xi \geq \xi_{\sigma}+\eta\end{cases}
\end{aligned}
$$

uniformly.

Here and after, denote $U^{\varepsilon}, \rho^{\varepsilon}$ as $U, \rho$ when there is no confusion.

Proof. Take $\xi_{3}$ and let $\varepsilon$ be so small that $\xi_{\sigma}<\xi_{3}-\frac{\eta}{2}$. If $u_{i 1}>u_{i 2}(i=1,2, \ldots, n)$, then we get by integrating (3.2) twice on $\left[\xi_{3}, \xi\right]$ that

$$
u_{i}\left(\xi_{3}\right)-u_{i}(\xi)=-u_{i}^{\prime}\left(\xi_{3}\right) \int_{\xi_{3}}^{\xi} \exp \left(\int_{\xi_{3}}^{r} \frac{\rho(U \cdot N-s)}{\varepsilon} d s\right) d r
$$

Since when $\xi>\xi_{\sigma}$,

$$
\begin{aligned}
\rho(\xi) & =\rho_{2} \exp \left(\int_{\xi}^{+\infty} \frac{U^{\prime}(s) \cdot N}{U(s) \cdot N-s} d s\right) \\
& =\lim _{R \rightarrow+\infty} \rho_{2} \exp \left(\int_{\xi}^{R} \frac{(U(s) \cdot N-s)^{\prime}+1}{U(s) \cdot N-s} d s\right) \\
& \leq \lim _{R \rightarrow+\infty} \rho_{2} \frac{U_{2} \cdot N-R}{U(\xi) \cdot N-\xi} \exp \int_{\xi}^{R} \frac{d s}{U_{2} \cdot N-s} \\
& =\lim _{R \rightarrow+\infty} \rho_{2} \frac{U_{2} \cdot N-R}{U(\xi) \cdot N-\xi} \frac{U_{2} \cdot N-\xi}{U_{2} \cdot N-R} \\
& =\frac{\rho_{2}\left(U_{2} \cdot N-\xi\right)}{U(\xi) \cdot N-\xi}
\end{aligned}
$$

we have

$$
\rho(U \cdot N-\xi) \geq \rho_{2}\left(U_{2} \cdot N-\xi\right), \quad \xi \in\left(\xi_{\sigma},+\infty\right)
$$

Then

$$
\begin{aligned}
u_{i}\left(\xi_{3}\right)-u_{i}(\xi) & \geq-u_{i}^{\prime}\left(\xi_{3}\right) \int_{\xi_{3}}^{\xi} \exp \left(\int_{\xi_{3}}^{r} \frac{\rho_{2}\left(U_{2} \cdot N-s\right)}{\varepsilon} d s\right) d r \\
& =-u_{i}^{\prime}\left(\xi_{3}\right) \int_{\xi_{3}}^{\xi} \exp \left(\frac{\rho_{2}}{\varepsilon}\left(\left(U_{2} \cdot N-\xi_{3}\right)\left(r-\xi_{3}\right)-\frac{1}{2}\left(r-\xi_{3}\right)^{2}\right)\right) d r \\
& =-u_{i}^{\prime}\left(\xi_{3}\right) \int_{0}^{\xi-\xi_{3}} \exp \left(\frac{\rho_{2}}{\varepsilon}\left(\left(U_{2} \cdot N-\xi_{3}\right) r-\frac{1}{2} r^{2}\right)\right) d r
\end{aligned}
$$


Letting $\xi \rightarrow+\infty$, it follows that

$$
\begin{aligned}
u_{i 1}-u_{i 2} & \geq-u_{i}^{\prime}\left(\xi_{3}\right) \int_{0}^{+\infty} \exp \frac{\rho_{2}}{2 \varepsilon}\left(2\left(U_{2} \cdot N-\xi_{3}\right) r-r^{2}\right) d r \\
& \geq-u_{i}^{\prime}\left(\xi_{3}\right) \sqrt{\varepsilon} A_{3},
\end{aligned}
$$

where $A_{3}$ is a constant independent of $\varepsilon$. Thus

$$
\left|u_{i}^{\prime}\left(\xi_{3}\right)\right| \leq \frac{u_{i 1}-u_{i 2}}{\sqrt{\varepsilon} A_{3}} .
$$

So

$$
\left|u_{i}^{\prime}(\xi)\right| \leq \frac{u_{i 1}-u_{i 2}}{\sqrt{\varepsilon} A_{3}} \exp \left(\int_{\xi_{3}}^{\xi} \frac{\rho(U \cdot N-s)}{\varepsilon} d s\right) .
$$

Again notice that when $\xi>\xi_{3}$,

$$
\begin{aligned}
\rho(\xi) & =\rho_{2} \exp \left(\int_{\xi}^{+\infty} \frac{U^{\prime}(s) \cdot N}{U(s) \cdot N-s} d s\right) \\
& =\lim _{R \rightarrow+\infty} \rho_{2} \exp \left(\int_{\xi}^{R} \frac{(U(s) \cdot N-s)^{\prime}+1}{U(s) \cdot N-s} d s\right) \\
& \geq \lim _{R \rightarrow+\infty} \rho_{2} \frac{U_{2} \cdot N-R}{U(\xi) \cdot N-\xi} \exp \int_{\xi}^{R} \frac{d s}{U\left(\xi_{3}\right) \cdot N-s} \\
& =\lim _{R \rightarrow+\infty} \rho_{2} \frac{U_{2} \cdot N-R}{U(\xi) \cdot N-\xi} \frac{U\left(\xi_{3}\right) \cdot N-\xi}{U\left(\xi_{3}\right) \cdot N-R} \\
& =\frac{\rho_{2}\left(U\left(\xi_{3}\right) \cdot N-\xi\right)}{U(\xi) \cdot N-\xi}
\end{aligned}
$$

we have

$$
\rho(U \cdot N-\xi) \leq \rho_{2}\left(U\left(\xi_{3}\right) \cdot N-\xi\right), \quad \text { for } \xi>\xi_{3} .
$$

Then

$$
\left|u_{i}^{\prime}(\xi)\right| \leq \frac{u_{i 1}-u_{i 2}}{\sqrt{\varepsilon} A_{3}} \exp \left(-\frac{\rho_{2}}{\varepsilon} \int_{\xi_{3}}^{\xi}\left(s-U\left(\xi_{3}\right) \cdot N\right) d s\right),
$$

which implies that

$$
\lim _{\varepsilon \rightarrow 0^{+}} u_{i \xi}^{\varepsilon}(\xi)=0 \quad \text { uniformly for } \xi \geq \xi_{\sigma}+\eta, i=1,2, \ldots, n .
$$

Next, take $\xi_{4}$ such that $\xi>\xi_{4} \geq \xi_{\sigma}+\eta$. From

$$
u_{i}\left(\xi_{4}\right)-u_{i}(\xi)=-u_{i}^{\prime}\left(\xi_{4}\right) \int_{\xi_{4}}^{\xi} \exp \left(\int_{\xi_{4}}^{r} \frac{\rho(U \cdot N-s)}{\varepsilon} d s\right) d r
$$

we get

$$
\begin{aligned}
\left|u_{i}\left(\xi_{4}\right)-u_{i}(\xi)\right| & \leq\left|u_{i}^{\prime}\left(\xi_{4}\right)\right| \int_{\xi_{4}}^{\xi} \exp \left(\int_{\xi_{4}}^{r} \frac{-A_{4}}{2 \varepsilon} d s\right) d r \\
& \leq \frac{2 \varepsilon}{A_{4}}\left|u_{i}^{\prime}\left(\xi_{4}\right)\right|\left\{1-\exp \left(\frac{A_{4}}{2 \varepsilon}\left(\xi_{4}-\xi\right)\right)\right\}
\end{aligned}
$$


where $A_{4}=2 \rho_{2}\left(\xi_{4}-U\left(\xi_{4}\right) \cdot N\right)$. Letting $\xi \rightarrow+\infty$, we arrive at

$$
\left|u_{i}\left(\xi_{4}\right)-u_{i 2}\right| \leq \frac{2 \varepsilon}{A_{4}}\left|u_{i}^{\prime}\left(\xi_{4}\right)\right|
$$

which implies that

$$
\lim _{\varepsilon \rightarrow 0^{+}} u_{i}^{\varepsilon}(\xi)=u_{i 2} \quad \text { uniformly for } \xi \geq \xi_{\sigma}+\eta, i=1,2, \ldots, n .
$$

In a similar way as above, we obtain that

$$
\lim _{\varepsilon \rightarrow 0^{+}} u_{i \xi}^{\varepsilon}(\xi)=0 \text { and } \lim _{\varepsilon \rightarrow 0^{+}} u_{i}^{\varepsilon}(\xi)=u_{i 1}
$$

uniformly for $\xi \leq \xi_{\sigma}-\eta, i=1,2, \ldots, n$.

If $u_{i 1}<u_{i 2}(i=1,2, \ldots, n)$, then we can also obtain the same results analogously. The proof of Lemma 4.1 is completed.

Lemma 4.2. For any $\eta>0$,

$$
\lim _{\varepsilon \rightarrow 0^{+}} \rho^{\varepsilon}(\xi)= \begin{cases}\rho_{1} & \text { for } \xi<\xi_{\sigma}-\eta \\ \rho_{2} & \text { for } \xi>\xi_{\sigma}+\eta\end{cases}
$$

uniformly.

Proof. From (4.1) and (4.2) it follows that for any $\xi>\xi_{5}>\xi_{\sigma}+\eta$,

$$
\frac{\rho_{2}\left(U\left(\xi_{5}\right) \cdot N-\xi\right)}{U(\xi) \cdot N-\xi} \leq \rho(\xi) \leq \frac{\rho_{2}\left(U_{2} \cdot N-\xi\right)}{U(\xi) \cdot N-\xi}
$$

which yields

$$
\lim _{\varepsilon \rightarrow 0^{+}} \rho^{\varepsilon}(\xi)=\rho_{2} \quad \text { uniformly for } \xi>\xi_{\sigma}+\eta .
$$

The remaining part can be obtained similarly. This completes the proof.

In what follows, we study in more detail the limiting behavior of $\rho^{\varepsilon}$ in the neighborhood of $\xi=\xi_{\sigma}$ as $\varepsilon \rightarrow 0^{+}$. Denote

$$
\sigma=\xi_{\sigma}=\lim _{\varepsilon \rightarrow 0^{+}} \xi_{\sigma}^{\varepsilon}=\lim _{\varepsilon \rightarrow 0^{+}} U^{\varepsilon}\left(\xi_{\sigma}^{\varepsilon}\right) \cdot N=U(\sigma) \cdot N .
$$

Take $\xi_{1}<\sigma<\xi_{2}, \phi \in C_{0}^{\infty}\left[\xi_{1}, \xi_{2}\right]$ such that $\phi(\xi)=\phi(\sigma)$ for $\xi$ in a neighborhood $\Omega$ of $\xi=\sigma$ ( $\phi$ is called a sloping test function). When $0<\varepsilon<\varepsilon_{0}, \xi_{\sigma}^{\varepsilon} \in \Omega$.

From (3.1-3.2) we have

$$
\begin{gathered}
-\int_{\xi_{1}}^{\xi_{2}} \rho^{\varepsilon}\left(U^{\varepsilon} \cdot N-\xi\right) \phi^{\prime} d \xi+\int_{\xi_{1}}^{\xi_{2}} \rho^{\varepsilon} \phi d \xi=0, \\
-\int_{\xi_{1}}^{\xi_{2}} \rho^{\varepsilon}\left(U^{\varepsilon} \cdot N-\xi\right) U^{\varepsilon} \phi^{\prime} d \xi+\int_{\xi_{1}}^{\xi_{2}} \rho^{\varepsilon} U^{\varepsilon} \phi d \xi=\varepsilon \int_{\xi_{1}}^{\xi_{2}} U^{\varepsilon} \phi^{\prime \prime} d \xi .
\end{gathered}
$$

Concerning (4.4), since $\phi(\xi)$ is constant on the interval $\alpha_{1}<\sigma<\alpha_{2}$ for $\alpha_{1}, \alpha_{2} \in \Omega$, we have

$$
\int_{\xi_{1}}^{\xi_{2}} \rho^{\varepsilon}\left(U^{\varepsilon} \cdot N-\xi\right) \phi^{\prime} d \xi=\int_{\xi_{1}}^{\alpha_{1}} \rho^{\varepsilon}\left(U^{\varepsilon} \cdot N-\xi\right) \phi^{\prime} d \xi+\int_{\alpha_{2}}^{\xi_{2}} \rho^{\varepsilon}\left(U^{\varepsilon} \cdot N-\xi\right) \phi^{\prime} d \xi .
$$


It immediately follows from Lemmas 4.1 and 4.2 that

$$
\begin{aligned}
\lim _{\varepsilon \rightarrow 0^{+}} \int_{\xi_{1}}^{\xi_{2}} \rho^{\varepsilon}\left(U^{\varepsilon} \cdot N-\xi\right) \phi^{\prime} d \xi & =\int_{\xi_{1}}^{\alpha_{1}} \rho_{1}\left(U_{1} \cdot N-\xi\right) \phi^{\prime} d \xi+\int_{\alpha_{2}}^{\xi_{2}} \rho_{2}\left(U_{2} \cdot N-\xi\right) \phi^{\prime} d \xi \\
& =(-\sigma[\rho]+[\rho U] \cdot N) \phi(\sigma)+\int_{\xi_{1}}^{\xi_{2}} h(\xi-\sigma) \phi(\xi) d \xi
\end{aligned}
$$

where

$$
h(x)= \begin{cases}\rho_{1}, & x<0 \\ \rho_{2}, & x>0\end{cases}
$$

Returning to (4.4), we get

$$
\lim _{\varepsilon \rightarrow 0^{+}} \int_{\xi_{1}}^{\xi_{2}}\left(\rho^{\varepsilon}-h(\xi-\sigma)\right) \phi(\xi) d \xi=(-\sigma[\rho]+[\rho U] \cdot N) \phi(\sigma)
$$

for all sloping test functions $\phi \in C_{0}^{\infty}\left[\xi_{1}, \xi_{2}\right]$. For an arbitrary $\psi \in C_{0}^{\infty}\left[\xi_{1}, \xi_{2}\right]$, we take a sloping test function $\phi$ such that $\phi(\sigma)=\psi(\sigma)$ and

$$
\max _{\left[\xi_{1}, \xi_{2}\right]}|\phi-\psi|<\mu, \quad \mu>0 .
$$

Recalling that $\rho^{\varepsilon} \in L^{1}\left[\xi_{1}, \xi_{2}\right]$ uniformly, we find that

$$
\begin{aligned}
& \lim _{\varepsilon \rightarrow 0^{+}} \int_{\xi_{1}}^{\xi_{2}}\left(\rho^{\varepsilon}-h(\xi-\sigma)\right) \psi(\xi) d \xi \\
& =\lim _{\varepsilon \rightarrow 0^{+}} \int_{\xi_{1}}^{\xi_{2}}\left(\rho^{\varepsilon}-h(\xi-\sigma)\right) \phi(\xi) d \xi+O(\mu) \\
& =(-\sigma[\rho]+[\rho U] \cdot N) \phi(\sigma)+O(\mu) \\
& =(-\sigma[\rho]+[\rho U] \cdot N) \psi(\sigma)+O(\mu) .
\end{aligned}
$$

Sending $\mu \rightarrow 0$, we find that (4.6) holds for all $\phi \in C_{0}^{\infty}\left[\xi_{1}, \xi_{2}\right]$.

In a similar way, from (4.5) we can get

$$
\lim _{\varepsilon \rightarrow 0^{+}} \int_{\xi_{1}}^{\xi_{2}}\left(\rho^{\varepsilon} U^{\varepsilon}-H(\xi-\sigma)\right) \phi(\xi) d \xi=(-\sigma[\rho U]+[\rho U \otimes U] \cdot N) \phi(\sigma)
$$

for all $\phi \in C_{0}^{\infty}\left[\xi_{1}, \xi_{2}\right]$, where we have used the equality

$$
[\rho U(U \cdot N)]=[\rho U \otimes U] \cdot N
$$

and

$$
H(x)= \begin{cases}\rho_{1} U_{1}, & x<0 \\ \rho_{2} U_{2}, & x>0\end{cases}
$$

Thus, $\rho^{\varepsilon}$ and $\rho^{\varepsilon} U^{\varepsilon}$ converge in the weak star topology of $C_{0}^{\infty}\left(R^{1}\right)$, and the limit functions are all sums of a step function and a Dirac delta function with strengths which are $-\sigma[\rho]+[\rho U] \cdot N$ and $-\sigma[\rho U]+[\rho U \otimes U] \cdot N$, respectively. 
If we take the test function in the $i$-th equation in $(4.5)$ as $\frac{\phi}{\tilde{u}_{i}^{\varepsilon}+\nu}(i=1,2, \ldots, n)$, where $\tilde{u}_{i}^{\varepsilon}$ is a modified function satisfying $u_{i}^{\varepsilon}(\sigma)$ in $\Omega$ and $u_{i}^{\varepsilon}$ outside $\Omega$, and let $\nu \rightarrow 0$, then we can find the other formula as follows:

$$
\lim _{\varepsilon \rightarrow 0^{+}} \int_{\xi_{1}}^{\xi_{2}}\left(\rho^{\varepsilon}-h(\xi-\sigma)\right) \phi(\xi) d \xi \cdot u_{i}(\sigma)=\left(-\sigma\left[\rho u_{i}\right]+\left[\rho u_{i}(U \cdot N)\right]\right) \phi(\sigma),
$$

where $\phi \in C_{0}^{\infty}\left[\xi_{1}, \xi_{2}\right], i=1,2, \ldots, n$. Combining with these formulas, we have

$$
\lim _{\varepsilon \rightarrow 0^{+}} \int_{\xi_{1}}^{\xi_{2}}\left(\rho^{\varepsilon}-h(\xi-\sigma)\right) \phi(\xi) d \xi \cdot U(\sigma)=(-\sigma[\rho U]+[\rho U \otimes U] \cdot N) \phi(\sigma)
$$

for $\phi \in C_{0}^{\infty}\left[\xi_{1}, \xi_{2}\right]$. Then from (4.3), (4.6) and (4.9) we get

$$
\left\{\begin{array}{l}
U(\sigma) \cdot N=\sigma \\
(-\sigma[\rho]+[\rho U] \cdot N) \cdot U(\sigma)=-\sigma[\rho U]+[\rho U \otimes U] \cdot N .
\end{array}\right.
$$

It follows that

$$
\sigma(-\sigma[\rho]+[\rho U] \cdot N)=(-\sigma[\rho U]+[\rho U \otimes U] \cdot N) \cdot N
$$

i.e.,

$$
\sigma^{2}[\rho]-2 \sigma([\rho U] \cdot N)+([\rho U \otimes U] \cdot N) \cdot N=0 .
$$

When $\rho_{1} \neq \rho_{2}$, we solve

$$
\sigma=\frac{[\rho U] \cdot N \pm \sqrt{\rho_{1} \rho_{2}}([U] \cdot N)}{[\rho]}
$$

i.e.,

$$
\sigma_{-}=\frac{\left(\sqrt{\rho_{1}} U_{1}+\sqrt{\rho_{2}} U_{2}\right) \cdot N}{\sqrt{\rho_{1}}+\sqrt{\rho_{2}}}, \quad \sigma_{+}=\frac{\left(\sqrt{\rho_{1}} U_{1}-\sqrt{\rho_{2}} U_{2}\right) \cdot N}{\sqrt{\rho_{1}}-\sqrt{\rho_{2}}} .
$$

Here we have used the equality

$$
([\rho U \otimes U] \cdot N) \cdot N=[\rho U(U \cdot N)] \cdot N=\left[(U \cdot N)^{2}\right] .
$$

Because of $U_{2} \cdot N<\sigma<U_{1} \cdot N$, we take

$$
\sigma=\sigma_{-}=\frac{\left(\sqrt{\rho_{1}} U_{1}+\sqrt{\rho_{2}} U_{2}\right) \cdot N}{\sqrt{\rho_{1}}+\sqrt{\rho_{2}}} .
$$

Corresponding to $\sigma=\sigma_{-}$, from (4.3) it follows that

$$
U_{\delta}=U(\sigma)=\frac{\sqrt{\rho_{1}} U_{1}+\sqrt{\rho_{2}} U_{2}}{\sqrt{\rho_{1}}+\sqrt{\rho_{2}}} .
$$

When $\rho_{1}=\rho_{2}$, from (4.11), we solve

$$
\sigma=\frac{\left(U_{1}+U_{2}\right) \cdot N}{2} \text { and } U_{\delta}=\frac{U_{1}+U_{2}}{2} .
$$

Therefore, the strengths of the Dirac delta functions are $\sqrt{\rho_{1} \rho_{2}}([U] \cdot N)$ and $\sqrt{\rho_{1} \rho_{2}}([U]$. $N) \sigma$, respectively, where $\sigma$ is as defined in (4.13). Then we have 
Theorem 4.3. Suppose that $\left(\rho^{\varepsilon}, U^{\varepsilon}\right)$ is a self-similar solution of (1.8) and (2.1) (i.e., $(3.1-3.3))$, and $[U] \cdot N>0$. Then

$$
\lim _{\varepsilon \rightarrow 0^{+}} U^{\varepsilon}(t, X)= \begin{cases}U_{1}, & X \cdot N<\sigma t, \\ U_{\delta}, & X \cdot N=\sigma t, \\ U_{2}, & X \cdot N>\sigma t,\end{cases}
$$

and $\rho^{\varepsilon}$ and $\rho^{\varepsilon} U^{\varepsilon}$ converge in the weak star topology of $C_{0}^{\infty}\left(R^{1}\right)$, the limit functions are all sums of a step function and a Dirac delta function supported on $X \cdot N=\sigma t$ with strengths $\sqrt{\rho_{1} \rho_{2}}([U] \cdot N)$ and $\sqrt{\rho_{1} \rho_{2}}([U] \cdot N) \sigma$, respectively, where $\sigma$ is as in (4.13) and $U_{\delta}$ as in (4.14).

Theorem 4.3 exhibits the structure of delta-shocks of (1.1). Also, we have seen the difference between the strength and (1.3), which is due to the result of self-similarity of viscosity.

CASE 2. $[U] \cdot N<0$.

LEMMA 4.4. For any $\eta>0$,

$$
\begin{gathered}
\lim _{\varepsilon \rightarrow 0^{+}} U_{\xi}^{\varepsilon}(\xi)=O, \quad \text { for } \xi \leq U_{1} \cdot N-\eta \text { or } \xi \geq U_{2} \cdot N+\eta, \\
\lim _{\varepsilon \rightarrow 0^{+}}\left(\rho^{\varepsilon}, U^{\varepsilon}\right)(\xi)= \begin{cases}\left(\rho_{1}, U_{1}\right), & \text { for } \xi<U_{1} \cdot N-\eta, \\
\operatorname{Vac}, & \text { for } U_{1} \cdot N-\eta \leq \xi \leq U_{2} \cdot N+\eta, \\
\left(\rho_{2}, U_{2}\right), & \text { for } \xi>U_{2} \cdot N+\eta,\end{cases}
\end{gathered}
$$

uniformly.

Proof. The proof of this lemma is basically similar to that of Lemmas 4.1-4.2. We present it just for completeness.

Take $\xi_{3}=U_{2} \cdot N+\eta$. Integrating (3.2) twice on $\left[\xi_{3}, \xi\right]$, we get if $u_{i 1}<u_{i 2}(i=$ $1,2, \ldots, n)$,

$$
\begin{aligned}
u_{i}(\xi)-u_{i}\left(\xi_{3}\right) & =u_{i}^{\prime}\left(\xi_{3}\right) \int_{\xi_{3}}^{\xi} \exp \left(\int_{\xi_{3}}^{r} \frac{\rho(U \cdot N-s)}{\varepsilon} d s\right) d r \\
& >u_{i}^{\prime}\left(\xi_{3}\right) \int_{\xi_{3}}^{\xi} \exp \left(\int_{\xi_{3}}^{r} \frac{\rho_{2}\left(U_{1} \cdot N-s\right)}{\varepsilon} d s\right) d r \\
& =u_{i}^{\prime}\left(\xi_{3}\right) \int_{\xi_{3}}^{\xi} \exp \left(\frac{\rho_{2}}{\varepsilon}\left(\left(U_{1} \cdot N-\xi_{3}\right)\left(r-\xi_{3}\right)-\frac{1}{2}\left(r-\xi_{3}\right)^{2}\right)\right) d r \\
& =u_{i}^{\prime}\left(\xi_{3}\right) \int_{0}^{\xi-\xi_{3}} \exp \frac{\rho_{2}}{2 \varepsilon}\left(2\left(U_{1} \cdot N-\xi_{3}\right) r-r^{2}\right) d r .
\end{aligned}
$$

Letting $\xi \rightarrow+\infty$, it follows that

$$
\begin{aligned}
u_{i 2}-u_{i 1} & \geq u_{i}^{\prime}\left(\xi_{3}\right) \int_{0}^{+\infty} \exp \frac{\rho_{2}}{2 \varepsilon}\left(2\left(U_{1} \cdot N-\xi_{3}\right) r-r^{2}\right) d r \\
& \geq u_{i}^{\prime}\left(\xi_{3}\right) \sqrt{\varepsilon} A_{5}
\end{aligned}
$$


where $A_{5}$ is a constant independent of $\varepsilon$. Thus

$$
\left|u_{i}^{\prime}\left(\xi_{3}\right)\right| \leq \frac{u_{i 2}-u_{i 1}}{\sqrt{\varepsilon} A_{5}} .
$$

So

$$
\left|u_{i}^{\prime}\left(\xi_{3}\right)\right| \leq \frac{u_{i 2}-u_{i 1}}{\sqrt{\varepsilon} A_{5}} \exp \left(\int_{\xi_{3}}^{\xi} \frac{\rho(U \cdot N-s)}{\varepsilon} d s\right) .
$$

Note that for $\xi>\xi_{\sigma}$,

$$
\begin{aligned}
\rho(\xi) & =\rho_{2} \exp \left(\int_{\xi}^{+\infty} \frac{U^{\prime}(s) \cdot N}{U(s) \cdot N-s} d s\right) \\
& =\lim _{R \rightarrow+\infty} \rho_{2} \exp \left(\int_{\xi}^{+\infty} \frac{(U(s) \cdot N-s)^{\prime}+1}{U(s)-s} d s\right) \\
& \geq \lim _{R \rightarrow+\infty} \rho_{2} \frac{U_{2} \cdot N-R}{U(\xi) \cdot N-\xi} \exp \left(\int_{\varepsilon}^{+\infty} \frac{d s}{U_{2} \cdot N-s}\right) \\
& =\frac{\rho_{2}\left(U_{2} \cdot N-\xi\right)}{U(\xi) \cdot N-\xi}
\end{aligned}
$$

we have

$$
\rho(U(\xi) \cdot N-\xi) \leq \rho_{2}\left(U_{2} \cdot N-\xi\right), \quad \xi>\xi_{\sigma}
$$

Therefore

$$
\begin{aligned}
\left|u_{i}^{\prime}(\xi)\right| & \leq \frac{u_{i 2}-u_{i 1}}{\sqrt{\varepsilon} A_{5}} \exp \left(\int_{\xi_{3}}^{\xi} \frac{\rho_{2}\left(U_{2} \cdot N-s\right)}{\varepsilon} d s\right) \\
& =\frac{u_{i 2}-u_{i 1}}{\sqrt{\varepsilon} A_{5}} \exp \left(-\frac{\rho_{2}}{2 \varepsilon}\left(\left(U_{2} \cdot N-\xi\right)^{2}-\left(U_{2} \cdot N-\xi_{3}\right)^{2}\right)\right)
\end{aligned}
$$

which implies that

$$
\lim _{\varepsilon \rightarrow 0^{+}} u_{i \xi}^{\varepsilon}(\xi)=0, \quad \text { uniformly for } \xi \geq U_{2} \cdot N+\eta, i=1,2, \ldots, n .
$$

Next, we pick $\xi_{4}$ such that $\xi>\xi_{4} \geq U_{2} \cdot N+\eta$. From

$$
u_{i}(\xi)-u_{i}\left(\xi_{4}\right)=u_{i}^{\prime}\left(\xi_{4}\right) \int_{\xi_{4}}^{\xi} \exp \left(\int_{\xi_{4}}^{r} \frac{\rho(U \cdot N-s)}{\varepsilon} d s\right) d r
$$

we get

$$
\begin{aligned}
\left|u_{i}(\xi)-u_{i}\left(\xi_{4}\right)\right| & \leq\left|u_{i}^{\prime}\left(\xi_{4}\right)\right| \int_{\xi_{4}}^{\xi} \exp \left(\int_{\xi_{4}}^{r} \frac{\rho_{2}\left(U_{2} \cdot N-s\right)}{\varepsilon} d s\right) d r \\
& =\left|u_{i}^{\prime}\left(\xi_{4}\right)\right| \int_{\xi_{4}}^{\xi} \exp \left(\frac{\rho_{2}}{2 \varepsilon}\left(2\left(U_{2} \cdot N-\xi_{4}\right)\left(r-\xi_{4}\right)-\left(r-\xi_{4}\right)^{2}\right)\right) d r \\
& =\left|u_{i}^{\prime}\left(\xi_{4}\right)\right| \int_{0}^{\xi-\xi_{4}} \exp \frac{\rho_{2}}{2 \varepsilon}\left(2\left(U_{2} \cdot N-\xi_{4}\right) r-r^{2}\right) d r
\end{aligned}
$$

Letting $\xi \rightarrow+\infty$, it follows that

$$
\left|u_{i}\left(\xi_{4}\right)-u_{i 2}\right| \leq\left|u_{i}^{\prime}\left(\xi_{4}\right)\right| \sqrt{\varepsilon} A_{6},
$$


where $A_{6}$ is a constant independent of $\varepsilon$, which implies that

$$
\lim _{\varepsilon \rightarrow 0^{+}} u_{i}^{\varepsilon}(\xi)=u_{i 2}, \quad \text { uniformly for } \xi \geq U_{2} \cdot N+\eta, i=1,2, \ldots, n .
$$

Furthermore, from Lemma 3.2(ii) and (4.16) we have that for $\xi>U_{2} \cdot N+\eta$,

$$
\rho_{2} \geq \rho(\xi) \geq \frac{\rho_{2}\left(U_{2} \cdot N-\xi\right)}{U(\xi) \cdot N-\xi} \rightarrow \rho_{2}, \quad \text { as } \varepsilon \rightarrow 0^{+} .
$$

Therefore,

$$
\lim _{\varepsilon \rightarrow 0^{+}} \rho^{\varepsilon}(\xi)=\rho_{2}, \quad \text { uniformly for } \xi>U_{2} \cdot N+\eta .
$$

In a similar way as above, we obtain that

$$
\lim _{\varepsilon \rightarrow 0^{+}} u_{i \xi}^{\varepsilon}=0 \text { and } \lim _{\varepsilon \rightarrow 0^{+}} u_{i}^{\varepsilon}(\xi)=u_{i 1}
$$

uniformly for $\xi \leq U_{1} \cdot N-\eta, i=1,2, \ldots, n$, as well as

$$
\lim _{\varepsilon \rightarrow 0^{+}} \rho^{\varepsilon}(\xi)=\rho_{1}, \quad \text { uniformly for } \xi<U_{1} \cdot N-\eta \text {. }
$$

Now let us consider the limit solution on $\left[U_{1} \cdot N, U_{2} \cdot N\right]$. Set

$$
F(\xi)=U(\xi) \cdot N-\xi
$$

Then from Lemma 3.2(ii) we get

$$
F^{\prime}(\xi)=U^{\prime}(\xi) \cdot N-1 \leq U^{\prime}\left(\xi_{\sigma}\right) \cdot N-1 \leq 0,
$$

where $\xi_{\sigma}$ satisfies $U\left(\xi_{\sigma}\right) \cdot N=\xi_{\sigma}, \xi \in\left[U_{1} \cdot N, U_{2} \cdot N\right]$. Hence

$$
F\left(U_{2} \cdot N+\eta\right) \leq F(\xi) \leq F\left(U_{1} \cdot N-\eta\right)
$$

namely

$$
U\left(U_{2} \cdot N+\eta\right) \cdot N-\left(U_{2} \cdot N+\eta\right) \leq U(\xi) \cdot N-\xi \leq U\left(U_{1} \cdot N-\eta\right) \cdot N-\left(U_{1} \cdot N-\eta\right)
$$

which yields

$$
-\eta \leq \lim _{\varepsilon \rightarrow 0^{+}}(U(\xi) \cdot N-\xi) \leq \eta .
$$

Since $\eta$ is arbitrary, we conclude that

$$
\lim _{\varepsilon \rightarrow 0^{+}}(U(\xi) \cdot N-\xi)=0 .
$$

This immediately shows that

$$
\lim _{\varepsilon \rightarrow 0^{+}} \rho^{\varepsilon}(\xi)=0, \quad \text { uniformly for } \quad U_{1} \cdot N-\eta \leq \xi \leq U_{2} \cdot N+\eta .
$$

Then we obtain a vacuum solution with vacuum curve $\rho=0\left(U_{1} \cdot N \leq \xi \leq U_{2} \cdot N\right)$.

If $u_{i 1}>u_{i 2}(i=1,2, \ldots, n)$, then we can also obtain the same results analogously. The proof of Lemma 4.4 is completed.

Thus we have the following theorem. 
TheOREM 4.5. Suppose that $\left(\rho^{\varepsilon}, U^{\varepsilon}\right)$ is a self-similar solution of (1.8) and (2.1) (i.e., $(3.1-3.3))$, and $[U] \cdot N<0$. Then

$$
\lim _{\varepsilon \rightarrow 0^{+}}\left(\rho^{\varepsilon}, U^{\varepsilon}\right)(t, X)= \begin{cases}\left(\rho_{1}, U_{1}\right), & X \cdot N<\left(U_{1} \cdot N\right) t \\ \operatorname{Vac}, & \left(U_{1} \cdot N\right) t \leq X \cdot N \leq\left(U_{2} \cdot N\right) t \\ \left(\rho_{2}, U_{2}\right), & X \cdot N>\left(U_{2} \cdot N\right) t\end{cases}
$$

REMARK. For any normal $\tilde{N}$ of the initial discontinuity, we can let $\tilde{N}=k N$, where $k$ is a positive constant, $|N|^{2}=1$, and seek the self-similar solution

$$
(\rho, U)=(\rho, U)\left(\frac{X \cdot \tilde{N}}{t}\right)=(\rho, U)\left(\frac{k X \cdot N}{t}\right) .
$$

In the same way as above, we can also obtain Theorem 4.3 and Theorem 4.5. These show that the limit solutions are independent of the choice for $N$.

5. Conclusions. In this paper we complete a program, begun in [SZh, LZ], to study delta-shocks for zero-pressure gas dynamics. By using the generalized Rankine-Hugoniot relations to solve the Riemann problem with two pieces of constant initial data separated by a hyperplane in $n(n \geq 2)$ dimensions, we obtain the Riemann solution of the system, which contains two explicit structures, delta-shock and vacuum state, when the initial data belongs to BV space. The most interesting is to discover multidimensional planar delta-shock waves dependent upon a family of one parameter. Here it is noticeable that the case for $n=1$ is extremely special [LZ]. To guarantee the delta-shock be a unique entropy solution, we utilize a self-similar viscous vanishing method to choose it. Analogous effort receives success only for the 1-D system [SZh]. From Theorem 4.3 and Theorem 4.5 we have seen that the structure of solutions is the same as that of the above Riemann solution. We note that the structure is of self-similarity although the weight of the delta-shock is a linear function of $t$. Therefore, the structure of solutions of the system, especially the structure of delta-shock, is stable under the viscous perturbations. That is, we obtain the stability of delta-shocks for multidimensional zero-pressure gas dynamics.

6. Acknowledgment. The authors wish to thank Professor Tong Zhang, their advisor, for numerous enlightening discussions. The comments of the referee were most helpful in improving the presentation of this paper.

\section{REFERENCES}

[A] R. A. Adams, Sobolev Spaces, Pure and Applied Math., vol. 65, New York, Academic Press, 1975

[AH] R. K. Agarwal and D. W. Halt, A modified CUSP scheme in wave/particle split form for unstructured grid Euler flows, Frontiers of Computational Fluid Dynamics, edited by D. A. Caughey and M. M. Hafes, John Wiley and Sons, 1994

[B] F. Bouchut, On zero-pressure gas dynamics, Advances in kinetic theory and computing, Series on Advances in Mathematics for Applied Sciences, Vol. 22, World Scientific, River Edge, NJ, 1994, pp. $171-190$

[BG] Y. Brenier and E. Grenier, Sticky particles and scalar conservation laws, SIAM J. Numer. Anal. 35, 2317-2328 (1998) 
[D] C. M. Dafermos, Solution of the Riemann problem for a class of hyperbolic systems of conservation laws by viscosity method, Arch. Rational Mech. Anal. 52, 1-9 (1973)

[ERS] W. E, Yu. G. Rykov, and Ya. G. Sinai, Generalized variational principles, global weak solutions and behavior with random initial data for systems of conservation laws arising in adhesion particle dynamics, Comm. Math. Phys. 177, 349-380 (1996)

[J] K. T. Joseph, A Riemann problem whose viscosity solutions contain delta-measures, Asymptotic Analysis 7, 105-120 (1993)

[Ko] D. J. Korchinski, Solutions of a Riemann problem for a $2 \times 2$ system of conservation laws possessing classical solutions, Adelphi University Thesis, 1977

[KK] B. L. Keyfitz and H. C. Kranzer, A viscosity approximation to system of conservation laws with no classical Riemann solution in Nonlinear Hyperbolic Problems, Lecture Notes in Mathematics, Vol. 1042, Springer-Verlag, Berlin/New York, 1989

[La] P. D. Lax, Hyperbolic systems of conservation laws and the mathematical theory of shock waves, SIAM, Philadelphia, 1973

[Le] P. Le Floch, An existence and uniqueness result for two nonstrictly hyperbolic systems, in Nonlinear Evolution Equations that Change Type, IMA 27 in Mathematics and its Applications, Springer-Verlag, 1990

[LC] Y. Li and Y. Cao, Large particle difference method with second order accuracy in gas dynamics, Scientific Sinica (A) 28, 1024-1035 (1985)

[LL] J. Li and W. Li, The Riemann problem for the zero-pressure flow in gas dynamics, Progress in Natural Sciences, to appear

[LZ] J. Li and T. Zhang, Generalized Rankine-Hugoniot relations of delta-shocks in solutions of transportation equations, Advances in Nonlinear Partial Differential Equations and Related Areas, World Sci. Publishing, River Edge, NJ, 1998

[M-Z] A. Majda, G. Majda, and Y. Zheng, Concentrations in the one-dimensional Vlasov-Poisson equations I: Temporal development and non-unique weak solutions in the single component case, p. 290, equation (5.12), Physica D, 74, 268-300 (1994)

[SZe] S. F. Shandarin and Ya. B. Zeldovich, The large-scale structure of the universe: Turbulence, intermittency, structures in a self-gravitating medium, Rev. Mod. Phys. 61, 185-220 (1989)

[SZh] W. Sheng and T. Zhang, The Riemann problem for the transportation equations in gas dynamics, Mem. Amer. Math. Soc. 137 (1999)

[TZ] D. Tan and T. Zhang, Two-dimensional Riemann problem for a hyperbolic system of nonlinear conservation laws (I): Four-J cases, J. Differential Equations 111, 203-254 (1994)

[TZZ] D. Tan, T. Zhang, and Y. Zheng, Delta-shock waves as limits of vanishing viscosity for hyperbolic systems of conservation laws, J. Differential Equations 112, 1-32 (1994)

[ZZ] T. Zhang and Y. Zheng, Conjecture on the structure of solutions of the Riemann problem for two-dimensional gas dynamics systems, SIAM J. Math. 21, No. 3, 593-630 (1990) 